\title{
The Radio Spectral Energy Distribution and Star-formation Rate Calibration in Galaxies
}

\author{
F. S. Tabatabaei ${ }^{1,2,3}$, E. Schinnerer ${ }^{3}$, M. Krause ${ }^{4}$, G. Dumas ${ }^{3}$, S. Meidt ${ }^{3}$, A. Damas-Segovia ${ }^{4}$, R. Beck ${ }^{4}$, E. J. Murphy ${ }^{5}$, \\ D. D. Mulcahy ${ }^{6}$, B. Groves ${ }^{7}$, A. Bolatto ${ }^{8}$, D. Dale ${ }^{9}$, M. Galametz ${ }^{10}$, K. Sandstrom ${ }^{11}$, M. Boquien ${ }^{12}$, D. Calzetti ${ }^{13}$, R. C. Kennicutt ${ }^{14}$, \\ L. K. Hunt $^{15}$, I. De Looze ${ }^{16}$, and E. W. Pellegrini ${ }^{17}$ \\ ${ }^{1}$ Instituto de Astrofísica de Canarias, Vía Láctea S/N, E-38205 La Laguna, Spain; ftaba@iac.es \\ ${ }^{2}$ Departamento de Astrofísica, Universidad de La Laguna, E-38206 La Laguna, Spain \\ ${ }^{3}$ Max-Planck-Institut für Astronomie, Königstuhl 17, D-69117 Heidelberg, Germany \\ ${ }^{4}$ Max-Planck Institut für Radioastronomie, Auf dem Hügel 69, D-53121 Bonn, Germany \\ ${ }_{6}^{5}$ National Radio Astronomy Observatory, 520 Edgemont Road, Charlottesville, VA 22903, USA \\ ${ }^{6}$ Jodrell Bank Centre for Astrophysics, Alan Turing Building, School of Physics and Astronomy, \\ The University of Manchester, Oxford Road, Manchester, M13 9PL, UK \\ ${ }^{7}$ Research School of Astronomy and Astrophysics, Australian National University, Canberra, ACT 2611, Australia \\ 8 Department of Astronomy and Joint Space Institute, University of Maryland, MD 20642, USA \\ ${ }^{9}$ Department of Physics \& Astronomy, University of Wyoming, Laramie, WY 82071, USA \\ ${ }^{10}$ European Southern Observatory, Karl-Schwarzschild-Str. 2, D-85748 Garching, Germany \\ ${ }^{11}$ Center for Astrophysics and Space Sciences, Department of Physics, University of California, San Diego, 9500 Gilman Drive, La Jolla, CA 92093, USA \\ ${ }_{12}$ Unidad de Astronomía, Fac. Cs. Básicas, Universidad de Antofagasta, Avda. U. de Antofagasta 02800, Antofagasta, Chile \\ ${ }_{13}^{13}$ Department of Astronomy, University of MassachusettsAmherst, Amherst, MA 01003, USA \\ ${ }^{14}$ Institute of Astronomy, University of Cambridge, Madingley Road, Cambridge CB3 OHA, UK \\ ${ }^{15}$ INAF-Osservatorio Astrofisico di Arcetri, Largo E. Fermi 5, I-50125, Firenze, Italy \\ ${ }^{16}$ Department of Physics \& Astronomy, University College London, Gower Place, London WC1E 6BT, UK \\ ${ }^{17}$ Zentrum für Astronomie der Universität Heidelberg, Institut für Theoretische Astrophysik, Albert-Ueberle-Str. 2, D-69120 Heidelberg, Germany \\ Received 2016 May 9; revised 2016 December 5; accepted 2016 December 6; published 2017 February 21
}

\begin{abstract}
We study the spectral energy distribution (SED) of the radio continuum (RC) emission from the Key Insight in Nearby Galaxies Emitting in Radio (KINGFISHER) sample of nearby galaxies to understand the energetics and origin of this emission. Effelsberg multi-wavelength observations at 1.4, 4.8, 8.4, and $10.5 \mathrm{GHz}$ combined with archive data allow us, for the first time, to determine the mid-RC (1-10 GHz, MRC) bolometric luminosities and further present calibration relations versus the monochromatic radio luminosities. The 1-10 GHz radio SED is fitted using a Bayesian Markov Chain Monte Carlo technique leading to measurements for the nonthermal spectral index $\left(S_{\nu} \sim \nu^{-\alpha_{\mathrm{nt}}}\right)$ and the thermal fraction $\left(f_{\mathrm{th}}\right)$ with mean values of $\alpha_{\mathrm{nt}}=0.97 \pm 0.16(0.79 \pm 0.15$ for the total spectral index) and $f_{\text {th }}=(10 \pm 9) \%$ at $1.4 \mathrm{GHz}$. The MRC luminosity changes over $\sim 3$ orders of magnitude in the sample, $4.3 \times 10^{2} L_{\odot}<$ MRC $<3.9 \times 10^{5} L_{\odot}$. The thermal emission is responsible for $\sim 23 \%$ of the MRC on average. We also compare the extinction-corrected diagnostics of the star-formation rate (SFR) with the thermal and nonthermal radio tracers and derive the first star-formation calibration relations using the MRC radio luminosity. The nonthermal spectral index flattens with increasing SFR surface density, indicating the effect of the star-formation feedback on the cosmic-ray electron population in galaxies. Comparing the radio and IR SEDs, we find that the FIR-to-MRC ratio could decrease with SFR, due to the amplification of the magnetic fields in starforming regions. This particularly implies a decrease in the ratio at high redshifts, where mostly luminous/starforming galaxies are detected.
\end{abstract}

Key words: galaxies: ISM - galaxies: star formation - infrared: galaxies - radio continuum: galaxies - surveys

Supporting material: machine-readable tables

\section{Introduction}

The use of the radio continuum (RC) emission as an extinction-free tracer of star formation in galaxies was first suggested by the tight empirical radio-infrared (IR) correlation, extending to more than four orders of magnitude in luminosity (see Condon et al. 2002, and references therein). However, some authors have raised the possibility of conspiracy of several factors as the cause of the radio-IR correlation (Bell 2003; Lacki et al. 2010). More direct studies of the radio emission properties at several frequencies are needed to understand the origins, energetics, and the thermal and nonthermal processes producing the RC emission observed in galaxies. Star-forming regions as the most powerful source of the RC emission are directly evident in the resolved maps of not only the thermal free-free emission but also the nonthermal synchrotron emission in nearby galaxies (Tabatabaei et al. 2007, 2013a, 2013c; Heesen et al. 2014;
Srivastava et al. 2014). This is understandable because massive star-formation activities like supernova explosions, their shocks, and remnants increase the number density of high-energy cosmic-ray electrons (CREs) and/or accelerate them, on one hand, and amplify the turbulent magnetic field strength, on the other hand. The net effect of these processes is a strong nonthermal emission in or around star-forming regions. These maps also show that extended structures in non-star-forming regions emit $\mathrm{RC}$, as well, but at lower intensities than in starforming regions. How these various sources/emission shape the $\mathrm{RC}$ spectrum globally and locally is a pressing question today.

Studying the spectral energy distribution (SED) provides significant information on the origin, energetics, and physics of the electromagnetic radiation in general. The shapes of the SEDs usually reflect the radiation laws and their parameters such as power-law energy index or emissivity index as well as 
physical phenomenon affecting those parameters like cooling/ heating mechanisms in the interstellar medium. Integrating the SEDs determines the total energy output of a source over a certain frequency range, which is a useful parameter to compare the energetics from different regimes of the electromagnetic radiation. Comparing the SEDs at different regimes (like in the radio and IR) provides key insights on the origin/ nature of the emission and general factors setting their energy balance. To date, the IR SEDs of various astrophysical objects have been dissected thanks to the coherent and simultaneous observations at several bands/frequencies with space telescopes like IRAS, ISO, Spitzer, and Herschel. In radio, however, most of the surveys have targeted a single radio frequency/band (mainly $1.4 \mathrm{GHz}$ ) with different sensitivities/ resolutions/observational instruments prohibiting a coherent (i.e., consistent in terms of performance/observations, targets and selection limits) radio-SED analysis for galaxy samples. This has been mainly because of a simple assumption under which the nonthermal radio spectrum has a fixed power-law index of $\alpha_{\mathrm{nt}} \sim 0.8$ (for $S \sim \nu^{-\alpha_{\mathrm{nt}}}$ ). However, this assumption cannot explain either the resolved spectra of galaxies (e.g., Tabatabaei et al. 2007, 2013c) or the integrated spectra (Duric et al. 1988; Marvil et al. 2015).

The radio SED of galaxies can be divided into two main domains: the nonthermal domain at $\nu \lesssim 10 \mathrm{GHz}$ and the thermal domain at frequencies of $10-20 \mathrm{GHz}<\nu<100 \mathrm{GHz}$. The aging of CREs and the thermal free-free absorption could cause curvature or flattening of the nonthermal SEDs. Such a flattening and curvature mostly occurs at low frequencies of $\nu<1 \mathrm{GHz}$ in galaxies (e.g., Condon 1992; Adebahr et al. 2013; Mulcahy et al. 2014; Marvil et al. 2015). In the mid-frequency range (MRC) of $1<\nu<10 \mathrm{GHz}$, the synchrotron power-law index faces minimal variations with frequency, on one hand, and the $\mathrm{RC}$ has the least contribution from spinning dust, on the other hand. Hence, the power-law SED (which is expected if the cooling and aging of CREs occur in a clumpy ISM, Basu et al. 2015a) could be optimally constrained in this frequency range. Extrapolating the $1-10 \mathrm{GHz}$ SEDs toward lower frequencies would then provide a basis to obtain the amplitude of the various effects causing possible flattening or curvature of the nonthermal spectrum. Toward higher frequencies, the extrapolations would help uncover potential contribution from anomalous dust emission.

This paper presents a coherent multi-band survey of the $1-10 \mathrm{GHz}$ SEDs in a statistically meaningful nearby galaxy sample, the Key Insights on Nearby Galaxies (KINGFISH; a Far-IR Survey with Herschel, Kennicutt et al. 2011) sample, providing a wide range in star-formation rate (SFR), morphology, and mass with the $100 \mathrm{~m}$ Effelsberg telescope. The KINGFISH sample is ideally suited to characterize the radio SEDs with respect to their IR SEDs that have been presented in Dale et al. (2012). Without any pre-assumption about $\alpha_{\mathrm{nt}}$, the true range of radio SED parameters are searched by means of the Bayesian Markov Chain Monte Carlo (MCMC) technique. The dependence of the radio SED parameters on the SFR are then studied using the measurements already available for the KINGFISH sample (Kennicutt et al. 2011).

The thermal and nonthermal radio fluxes separated using the SED modeling allow us to estimate the SFR using the basic thermal/nonthermal radio SFR calibration relations presented in Murphy et al. (2011) and to compare the radio SFRs with other extinction-free SFR tracers.
The paper is organized as follows. After presenting the observations and the data (Section 2), we describe the SED modeling and present the results (Section 3). In Section 4, we introduce the MRC bolometric SED and determine the contribution of the standard radio bands. The calibration relations based on the radio emission are presented in Section 5. The decomposed nonthermal emission allows estimation of the equipartition magnetic field strength for the sample (Section 6). We then discuss the results (Section 7) and summarize our findings (Section 8).

\section{Data}

\subsection{Radio Observations and Data Reduction}

The KINGFISH sample consists of 61 nearby galaxies of different morphological types. From this sample, we selected all galaxies with declinations $\geqslant-21^{\circ}$ and named this subsample KINGFISHER (KINGFISH galaxies Emitting in Radio). These galaxies can be observed with the Effelsberg $100 \mathrm{~m}$ single-dish telescope to obtain global measurements of the RC at $20 \mathrm{~cm}$, $6 \mathrm{~cm}$, and $3.6 \mathrm{~cm}$. ${ }^{18}$ About 50 galaxies fulfill this criterion. The non-KINGFISH galaxy, M51, is also included in this study. We observed 35 of these galaxies at $6 \mathrm{~cm}, 10$ galaxies at $20 \mathrm{~cm}$, and 7 at $3.6 \mathrm{~cm}$ to complete already existing archival data during four observation runs listed in Table 2. Table 1 summarizes some KINGFISHER sample properties, and Table 3 summarizes the new Effelsberg observations.

\subsubsection{The $6 \mathrm{~cm}$ Observations}

At $6 \mathrm{~cm}$, the beam size of the Effelsberg telescope is 2.5 , which is comparable to the optical sizes of some of our targets. Two modes of observation were used, depending on the size of the target. The 19 smaller and fainter galaxies were observed in the cross-scan mode (point-source observations). In this mode, the objects were observed in $20^{\prime}$ long scans in azimuth and in elevation with a velocity of $30^{\prime}$ minutes $^{-1}$. For galaxies with $20 \mathrm{~cm}$ flux densities lower than $\sim 10 \mathrm{mJy}$ and those not detected in NVSS (11 galaxies), 30 cross-scans were used leading to an on-source time of 30 minutes per target. For the other five bright compact galaxies, only 10 cross-scans ( $\sim 10$ minutes per target) were used. The remaining 16 galaxies were observed in the mapping mode. The Effelsberg maps at $6 \mathrm{~cm}$ are scanned in the azimuthal direction with a two-horn secondary-focus system, using software beam-switching (Emerson et al. 1979), corrected for baselevel, and transformed into the R.A., decl. coordinate system. We obtained maps of $18^{\prime} \times 10^{\prime}$ (grid size of $60^{\prime \prime}$ ) for the five sources with optical sizes of $D<7^{\prime}$, and $26^{\prime} \times 18^{\prime}$ maps for the remaining 10 galaxies. A map size of $28^{\prime} \times 20^{\prime}$ was used for NGC 5055 . With 20 coverages per target, we achieved a $0.3 \mathrm{mJy} /$ beam rms noise. The total on-source observing times are 200 minutes $(=20 \times 10$ minutes $)$ for the $18^{\prime} \times 10^{\prime}$ maps, 320 minutes $(=20 \times 16$ minutes $)$ for the $26^{\prime} \times 18^{\prime}$ maps, and 480 minutes $(=20 \times 24$ minutes $)$ for NGC 5055 .

The pointed observations were reduced using the program package Toolbox. ${ }^{19}$ The resulting fluxes were then corrected for opacity and pointing offsets. After correcting for various effects including the gain curve, the conversion from Kelvin to Jansky

\footnotetext{
${ }^{18}$ Based on observations with the $100 \mathrm{~m}$ telescope of the Max-Planck-Institut für Radioastronomie at Effelsberg.

19 https://eff100mwiki.mpifr-bonn.mpg.de/
} 
Table 1

Basic Properties of the Galaxy Sample

\begin{tabular}{|c|c|c|c|c|c|c|c|c|c|}
\hline $\begin{array}{l}\text { Galaxy } \\
\text { Name }\end{array}$ & $\begin{array}{c}\text { R.A. } \\
(\mathrm{J} 2000)\end{array}$ & $\begin{array}{c}\text { Decl. } \\
(\mathrm{J} 2000)\end{array}$ & $\begin{array}{l}\text { Hubble } \\
\text { Type }^{\mathrm{a}}\end{array}$ & $\begin{array}{l}\text { Size }^{\mathrm{a}} \\
\left({ }^{\prime} \times{ }^{\prime}\right)\end{array}$ & $\begin{array}{c}\text { Inclination }^{\mathrm{b}} \\
\text { (degrees) }^{-}\end{array}$ & $\begin{array}{l}\text { Distance }^{c} \\
(\mathrm{Mpc})\end{array}$ & $\begin{array}{l}\text { Nuclear } \\
\text { Type }^{c}\end{array}$ & $\begin{array}{c}\log (\mathrm{TIR})^{\mathrm{d}} \\
\left(L_{\odot}\right)\end{array}$ & $\begin{array}{c}\mathrm{SFR}^{\mathrm{c}} \\
\left(M_{\odot} \mathrm{yr}^{-1}\right)\end{array}$ \\
\hline DDO053 & 083407.2 & +661054 & $\mathrm{Im}$ & $1.5 \times 1.3$ & 31 & 3.61 & $\ldots$ & 7.0 & 0.006 \\
\hline DDO154 & 125405.2 & +270855 & $\mathrm{IBm}$ & $3.0 \times 2.2$ & 66 & 4.3 & $\ldots$ & $6.9^{\mathrm{c}}$ & 0.002 \\
\hline DDO165 & 130624.8 & +674225 & $\mathrm{Im}$ & $3.5 \times 1.9$ & 61 & 4.57 & $\ldots$ & $7.0^{\mathrm{c}}$ & 0.002 \\
\hline HoI & 094032.3 & +711056 & IABm & $3.6 \times 3.0$ & 12 & 3.9 & $\cdots$ & 7.1 & 0.004 \\
\hline IC0342 & 034648.5 & +680546 & SABcd & $21.4 \times 20.9$ & 31 & 3.28 & SF & 10.1 & 1.87 \\
\hline IC2574 & 102821.2 & +682443 & $\mathrm{SABm}$ & $13.2 \times 5.4$ & 53 & 3.79 & SF & 8.3 & 0.057 \\
\hline M81DwB & 100530.6 & +702152 & $\mathrm{Im}$ & $0.9 \times 0.6$ & 48 & 3.6 & $\cdots$ & 6.5 & 0.001 \\
\hline NGC 0337 & 005950.0 & -073441 & SBd & $2.9 \times 01.8$ & 52 & 19.3 & SF & 10.1 & 1.30 \\
\hline NGC 0584 & 013120.7 & -065204 & $\mathrm{E} 4$ & $4.2 \times 2.3$ & 58 & 20.8 & $\ldots$ & 8.8 & $\ldots$ \\
\hline NGC 0628 & 013641.7 & +154701 & SAc & $10.5 \times 09.5$ & 25 & 7.2 & $\ldots$ & 9.9 & 0.68 \\
\hline NGC 0855 & 021403.6 & +275239 & $\mathrm{E}$ & $2.6 \times 1.0$ & 70 & 9.73 & SF & 8.6 & $\ldots$ \\
\hline NGC 0925 & 022717.1 & +333445 & $\mathrm{SABd}$ & $10.5 \times 05.9$ & 66 & 9.12 & SF & 9.7 & 0.54 \\
\hline NGC 1266 & 031600.7 & -022538 & SB0 & $1.5 \times 01.0$ & 32 & 30.6 & $\mathrm{AGN}$ & 10.4 & $\cdots$ \\
\hline NGC 1377 & 033639.1 & -205408 & So & $1.8 \times 0.9$ & 62 & 24.6 & $\cdots$ & 10.1 & 1.86 \\
\hline NGC 1482 & 035438.9 & -203008 & SA0 & $2.5 \times 01.4$ & 57 & 22.6 & SF & 10.6 & 3.57 \\
\hline NGC 2146 & 061837.7 & +782125 & Sbab & $6.0 \times 03.4$ & 57 & 17.2 & SF & 11.0 & 7.94 \\
\hline NGC 2798 & 091722.9 & +420000 & $\mathrm{SBa}$ & $2.6 \times 01.0$ & 68 & 25.8 & $\mathrm{SF} / \mathrm{AGN}$ & 10.6 & 3.38 \\
\hline NGC 2841 & 092202.6 & +505835 & $\mathrm{SAb}$ & $8.1 \times 3.5$ & 74 & 14.1 & $\mathrm{AGN}$ & 10.1 & 2.45 \\
\hline NGC 2976 & 094715.3 & +675500 & SAc & $5.9 \times 2.7$ & 65 & 3.55 & SF & 8.9 & 0.082 \\
\hline NGC 3049 & 095449.6 & +09 1617 & SBab & $2.2 \times 1.4$ & 61 & 19.2 & SF & 9.5 & 0.61 \\
\hline NGC 3077 & 100319.1 & +684402 & IOpec & $5.4 \times 4.5$ & 33 & 3.83 & SF & 8.9 & 0.094 \\
\hline NGC 3184 & 101816.9 & +412528 & SABcd & $7.4 \times 6.9$ & 16 & 11.7 & $\mathrm{SF}$ & 10.0 & 0.66 \\
\hline NGC 3190 & 101805.6 & +214956 & SAap & $4.4 \times 1.5$ & 73 & 19.3 & $\mathrm{AGN}$ & 9.9 & 0.38 \\
\hline NGC 3198 & 101954.9 & +453259 & $\mathrm{SBc}$ & $8.5 \times 3.3$ & 72 & 14.1 & SF & 10.0 & 1.01 \\
\hline NGC 3265 & 103106.7 & +284748 & $\mathrm{E}$ & $1.3 \times 1.0$ & 46 & 19.6 & SF & 9.4 & 0.38 \\
\hline NGC 3351 & 104357.7 & +114213 & $\mathrm{SBb}$ & $7.4 \times 5.0$ & 41 & 9.93 & SF & 9.9 & 0.58 \\
\hline NGC 3521 & 100548.6 & -000209 & $\mathrm{SABbc}$ & $11.0 \times 5.1$ & 73 & 11.2 & $\mathrm{SF} / \mathrm{AGN}$ & 10.5 & 1.95 \\
\hline NGC 3627 & 112014.9 & +125930 & $\mathrm{SABb}$ & $9.1 \times 4.2$ & 62 & 9.38 & $\mathrm{AGN}$ & 10.4 & 1.70 \\
\hline NGC 3773 & 113813.0 & +120644 & SA0 & $1.2 \times 1.0$ & 34 & 12.4 & $\mathrm{SF}$ & 8.8 & 0.16 \\
\hline NGC 3938 & 115249.4 & +440715 & SAc & $5.4 \times 4.9$ & 25 & 17.9 & SF & 10.3 & 1.77 \\
\hline NGC 4236 & 121642.1 & +692745 & SBdm & $21.9 \times 7.2$ & 72 & 4.45 & SF & 8.7 & 0.13 \\
\hline NGC 4254 & 121849.6 & +142459 & SAc & $5.4 \times 4.7$ & 29 & 14.4 & $\mathrm{SF} / \mathrm{AGN}$ & 10.6 & 3.92 \\
\hline NGC 4321 & 122254.8 & +154919 & $\mathrm{SABbc}$ & $7.4 \times 6.3$ & 32 & 14.3 & $\mathrm{AGN}$ & 10.5 & 2.61 \\
\hline NGC 4536 & 123427.0 & +021117 & $\mathrm{SABbc}$ & $7.6 \times 3.2$ & 67 & 14.5 & $\mathrm{SF} / \mathrm{AGN}$ & 10.3 & 2.17 \\
\hline NGC 4559 & 123557.7 & +275736 & SABcd & $10.7 \times 4.4$ & 66 & 6.98 & SF & 9.5 & 0.37 \\
\hline NGC 4569 & 123649.8 & +130947 & SABab & $9.5 \times 4.4$ & 64 & 9.86 & $\mathrm{AGN}$ & 9.7 & 0.29 \\
\hline NGC 4579 & 123743.5 & +114905 & $\mathrm{SABb}$ & $5.9 \times 4.7$ & 38 & 16.4 & $\mathrm{AGN}$ & 10.1 & 1.10 \\
\hline NGC 4594 & 123959.4 & -113723 & SAa & $8.7 \times 3.5$ & 69 & 9.08 & AGN & 9.6 & 0.18 \\
\hline NGC 4625 & 124152.6 & +411626 & SABmp & $2.2 \times 1.9$ & 30 & 9.3 & SF & 8.8 & 0.052 \\
\hline NGC 4631 & 124208.0 & +323229 & SBd & $15.5 \times 2.7$ & 83 & 7.62 & SF & 10.4 & 1.70 \\
\hline NGC 4725 & 125026.6 & +253003 & SABab & $10.7 \times 7.6$ & 45 & 11.9 & $\mathrm{AGN}$ & 9.9 & 0.44 \\
\hline NGC 4736 & 125053.1 & +410713 & SAab & $11.2 \times 9.1$ & 41 & 4.66 & $\mathrm{AGN}$ & 9.8 & 0.38 \\
\hline NGC 4826 & 125643.7 & +214100 & SAab & $10.0 \times 5.4$ & 65 & 5.27 & $\mathrm{AGN}$ & 9.6 & 0.26 \\
\hline NGC 5055 & 131549.3 & +420146 & $\mathrm{SAbc}$ & $12.6 \times 7.2$ & 59 & 7.94 & AGN & 10.3 & 1.04 \\
\hline NGC 5457 & 140312.6 & +542057 & SABcd & $28.8 \times 26.9$ & 18 & 6.7 & SF & 10.4 & 2.33 \\
\hline NGC 5474 & 140501.5 & +533945 & SAcd & $4.8 \times 4.3$ & 26 & 6.8 & SF & 8.8 & 0.091 \\
\hline NGC 5713 & 144011.5 & -001720 & SABbcp & $2.8 \times 2.5$ & 33 & 21.4 & SF & 10.5 & 2.52 \\
\hline NGC 5866 & 150629.5 & +554548 & S0 & $4.7 \times 01.9$ & 68 & 15.3 & $\mathrm{AGN}$ & 9.8 & 0.26 \\
\hline NGC 6946 & 203452.3 & +600914 & SABcd & $11.5 \times 9.8$ & 33 & 6.8 & SF & 10.5 & 7.12 \\
\hline NGC 7331 & 223704.1 & +342456 & $\mathrm{SAb}$ & $10.5 \times 03.7$ & 76 & 14.5 & AGN & 10.7 & 2.74 \\
\hline M51 & 132956.2 & +471350 & SAbc & $11.2 \times 6.9$ & $22^{\mathrm{e}}$ & $7.6^{\mathrm{f}}$ & $\mathrm{AGN}^{\mathrm{g}}$ & $10.5^{\mathrm{h}}$ & $5.0^{\mathrm{i}}$ \\
\hline
\end{tabular}

Notes.

a NASA Extragalactic Database.

${ }^{\mathrm{b}}$ Hunt et al. (2015) and references therein.

c Kennicutt et al. (2011) and references therein.

d Dale et al. (2012).

e Colombo et al. (2014).

${ }^{\mathrm{f}}$ Ciardullo et al. (1988).

g Matsushita et al. (2004).

h Rujopakarn et al. (2013).

${ }^{\mathrm{i}}$ Leroy et al. (2008).

(This table is available in machine-readable form.) 
Table 2

Effelsberg Projects

\begin{tabular}{ll}
\hline \hline Project Code & Observation Date \\
\hline $78-08$ & $2008 \mathrm{Dec}$ \\
$10-09$ & $2009 \mathrm{Dec}$ \\
$20-10$ & $2010 \mathrm{Apr}$ \\
$72-10$ & $2010 \mathrm{Dec} \& 2012 \mathrm{Mar}$ \\
\hline
\end{tabular}

was applied. The errors reported in Table 4 are uncertainties in fitting the cross-scan profiles.

\subsubsection{The $20 \mathrm{~cm}$ Observations}

No archival $20 \mathrm{~cm}$ data existed for 10 large galaxies $\left(>10^{\prime}\right.$ in extent). Hence, they were observed in our last run of observations (obs. code 20-10). The Effelsberg maps at $20 \mathrm{~cm}$ (and $3.6 \mathrm{~cm}$, see below) were scanned alternating in R. A. and decl. with one-horn systems and combined using the spatial-frequency weighting method by Emerson \& Graeve (1988). We obtained maps of $51^{\prime} \times 51^{\prime}$ for all these galaxies but NGC 5457 (M 101) for which a map of $90^{\prime} \times 90^{\prime}$ was obtained due to its large size. The beam size at $20 \mathrm{~cm}$ is $9 ! 15$ and we used a sampling of $3^{\prime}$ and a scanning velocity of $3^{\circ}$ minutes $^{-1}$. In order to reach the rms noise of about $6 \mathrm{mJy} /$ beam, we used four coverages of 12 minutes exposure time for each galaxy $(4 \times 26$ minutes for M 101).

\subsubsection{The $3.6 \mathrm{~cm}$ Observations}

At $3.6 \mathrm{~cm}$, we observed seven galaxies with a grid size of $30^{\prime \prime}$ and a scanning velocity of $20^{\prime}$ minutes $^{-1}$. With 13 coverages, we reached an $\mathrm{rms}$ noise of $0.5 \mathrm{mJy} /$ beam. The beam size at $3.6 \mathrm{~cm}$ is 1.5 . The map sizes are provided in Table 3.

The data reduction was performed using the NOD2 (and NOD3, P. Müller et al. 2017, in preparation) data reduction system (Haslam 1974). The maps were reduced using the program package Ozmapax. In order to remove scanning effects due to ground radiation, weather condition, and receiver instabilities, we applied the scanning removal program, Presse, of Sofue \& Reich (1979) in the mapping mode.

Throughout our observations, the quasars 3C48, 3C138, $3 \mathrm{C} 147$, and $3 \mathrm{C} 286$ were used as pointing, focus, and flux calibrators.

\subsection{Other Data}

The Effelsberg observations complement the already available radio data sets for the KINGFISH sample, which were mainly picked from the NVSS (Condon et al. 1998) at $20 \mathrm{~cm}$ and the Atlas of Shapley-Ames Galaxies at $2.8 \mathrm{~cm}$ (Niklas et al. 1995). Depending on the Galaxy/wavelength, we also used the archival Effelsberg radio data (see Table 4).

Herschel data were used to compare the radio and IR SEDs. The sample was observed with the Herschel Space Observatory as part of the KINGFISH project (Kennicutt et al. 2011) as described in detail in Dale et al. (2012) and Aniano et al. (2012). Although we used the calibrations by Dale et al. (2012), the newer calibrations reported by Hunt et al. (2015) change the luminosities by no more than $10 \%-15 \%$, within the $20 \%$ uncertainties quoted here. Table 4 lists the total IR
Table 3

Observing Modes and Covering Areas of the Galaxies Observed with the $100 \mathrm{~m}$ Telescope at the Three Wavelengths

\begin{tabular}{|c|c|c|c|}
\hline Galaxy & $3.6 \mathrm{~cm}$ & $6 \mathrm{~cm}$ & $20 \mathrm{~cm}$ \\
\hline DDO053 & $\cdots$ & pointed & $\ldots$ \\
\hline DDO154 & $\ldots$ & pointed & $\ldots$ \\
\hline DDO165 & $\cdots$ & pointed & $\cdots$ \\
\hline HoI & $\ldots$ & pointed & $\ldots$ \\
\hline IC2574 & $21^{\prime} \times 14^{\prime}$ & $\cdots$ & $\cdots$ \\
\hline M81DwB & $\ldots$ & pointed & $\ldots$ \\
\hline NGC 0337 & $10^{\prime} \times 10^{\prime}$ & pointed & $\ldots$ \\
\hline NGC 0584 & $\ldots$ & pointed & $\ldots$ \\
\hline NGC 0628 & $21^{\prime} \times 21^{\prime}$ & $26^{\prime} \times 18^{\prime}$ & $51^{\prime} \times 51^{\prime}$ \\
\hline NGC 0855 & $\ldots$ & pointed & $\ldots$ \\
\hline NGC 0925 & $\ldots$ & $26^{\prime} \times 18^{\prime}$ & $51^{\prime} \times 51^{\prime}$ \\
\hline NGC 1266 & $10^{\prime} \times 10^{\prime}$ & pointed & $\cdots$ \\
\hline NGC 1377 & $\cdots$ & pointed & $\cdots$ \\
\hline NGC 1482 & $10^{\prime} \times 10^{\prime}$ & pointed & $\ldots$ \\
\hline NGC 2146 & $\ldots$ & $18^{\prime} \times 10^{\prime}$ & $\ldots$ \\
\hline NGC 2798 & $10^{\prime} \times 10^{\prime}$ & pointed & $\ldots$ \\
\hline NGC 2841 & $\ldots$ & $26^{\prime} \times 18^{\prime}$ & $\ldots$ \\
\hline NGC 2976 & $\ldots$ & $26^{\prime} \times 18^{\prime}$ & $\ldots$ \\
\hline NGC 3049 & $\cdots$ & pointed & $\ldots$ \\
\hline NGC 3077 & $\cdots$ & $18^{\prime} \times 10^{\prime}$ & $\cdots$ \\
\hline NGC 3184 & $\ldots$ & $26^{\prime} \times 18^{\prime}$ & $\ldots$ \\
\hline NGC 3190 & $\cdots$ & pointed & $\ldots$ \\
\hline NGC 3198 & $\cdots$ & $26^{\prime} \times 18^{\prime}$ & $\ldots$ \\
\hline NGC 3265 & $\ldots$ & pointed & $\ldots$ \\
\hline NGC 3351 & $\cdots$ & $26^{\prime} \times 18^{\prime}$ & $\cdots$ \\
\hline NGC 3521 & $\cdots$ & $\ldots$ & $51^{\prime} \times 51^{\prime}$ \\
\hline NGC 3773 & $\cdots$ & pointed & $\cdots$ \\
\hline NGC 3938 & $\cdots$ & $26^{\prime} \times 18^{\prime}$ & $\ldots$ \\
\hline NGC 4559 & $\ldots$ & $26^{\prime} \times 18^{\prime}$ & $51^{\prime} \times 51^{\prime}$ \\
\hline NGC 4625 & $\cdots$ & pointed & \\
\hline NGC 4725 & $42^{\prime} \times 28^{\prime}$ & $26^{\prime} \times 18^{\prime}$ & $51^{\prime} \times 51^{\prime}$ \\
\hline NGC 4736 & $\ldots$ & $\cdots$ & $51^{\prime} \times 51^{\prime}$ \\
\hline NGC 4826 & $\cdots$ & $26^{\prime} \times 18^{\prime}$ & $51^{\prime} \times 51^{\prime}$ \\
\hline NGC 5055 & $\ldots$ & $28^{\prime} \times 20^{\prime}$ & $51^{\prime} \times 51^{\prime}$ \\
\hline NGC 5457 & $\cdots$ & $\cdots$ & $90^{\prime} \times 90^{\prime}$ \\
\hline NGC 5474 & $\cdots$ & pointed & $\cdots$ \\
\hline NGC 5713 & $\cdots$ & pointed & $\cdots$ \\
\hline NGC 5866 & $25^{\prime} \times 25^{\prime}$ & $18^{\prime} \times 10^{\prime}$ & $\cdots$ \\
\hline NGC 7331 & $\cdots$ & $26^{\prime} \times 18^{\prime}$ & $51^{\prime} \times 51^{\prime}$ \\
\hline
\end{tabular}

Note.The sizes refer to the areas of the observations. The center of the areas are the Galaxy centers. Pointed means cross-scan observing mode. See the text for details.

luminosities (TIR) based on Herschel PACS (Poglitsch et al. 2010) and SPIRE (Griffin et al. 2010) data.

We also used the Spitzer MIPS $24 \mu \mathrm{m}$ and the $\mathrm{H} \alpha$ data from SINGS (Kennicutt et al. 2003; Dale et al. 2007), and the FUV data from GALEX (Gil de Paz et al. 2007) as star-formation tracers.

\section{Radio SEDs}

Table 4 lists the integrated radio flux densities at various frequencies. The integration was performed up to the optical radius in order to be consistent with the measurements in the IR (Dale et al. 2012; see Section 7.3). The background estimate was determined far beyond the optical radius. In those cases where particularly bright background radio sources were present in the field, such sources were first interactively blanked from the 
Table 4

Radio Monochromatic Flux Densities and the MRC Luminosities

\begin{tabular}{|c|c|c|c|c|c|c|c|c|c|c|}
\hline $\begin{array}{l}\text { Galaxy } \\
\text { Name }\end{array}$ & $\begin{array}{c}S_{2.8 \mathrm{~cm}}^{10.7 \mathrm{GHz}} \\
(\mathrm{mJy})\end{array}$ & $\begin{array}{l}S_{3.6 \mathrm{GHz}}^{8.4 \mathrm{Gm}} \\
(\mathrm{mJy})\end{array}$ & $\begin{array}{l}S_{6 \mathrm{~cm}}^{5} \mathrm{GHz} \\
(\mathrm{mJy})\end{array}$ & $\begin{array}{c}S_{6.2 \mathrm{cmz}}^{4.8 \mathrm{cHz}} \\
(\mathrm{mJy})\end{array}$ & $\begin{array}{c}S_{8.1 \mathrm{Gm}}^{3.7 \mathrm{cHz}} \\
(\mathrm{mJy})\end{array}$ & $\begin{array}{c}S_{11.1 \mathrm{~cm}}^{2.7 \mathrm{GHz}} \\
(\mathrm{mJy})\end{array}$ & $\begin{array}{c}S_{20 \mathrm{~cm}}^{1.4 \mathrm{GHz}} \\
(\mathrm{mJy})\end{array}$ & $\begin{array}{c}S_{22 \mathrm{~cm}}^{1.36 \mathrm{GHz}} \\
(\mathrm{mJy})\end{array}$ & $\begin{array}{c}\log (\text { MRC }) \\
{\left[L_{\odot}\right]}\end{array}$ & $\begin{array}{r}\mathrm{B} \\
(\mu \mathrm{G})\end{array}$ \\
\hline DDO053 & $\ldots$ & $\ldots$ & $\ldots$ & $0.8 \pm 0.2^{\mathrm{a}}$ & $\ldots$ & $\ldots$ & $\ldots$ & $\ldots$ & $\ldots$ & $\ldots$ \\
\hline DDO154 & $\ldots$ & $\ldots$ & $\ldots$ & $<0.45^{\mathrm{a}}$ & $\ldots$ & $\ldots$ & $<1.5^{\mathrm{d}}$ & $\ldots$ & $\ldots$ & $\ldots$ \\
\hline DDO165 & $\ldots$ & $\ldots$ & $\ldots$ & $<0.43^{\mathrm{a}}$ & $\ldots$ & $\ldots$ & $<1.5^{\mathrm{d}}$ & $\ldots$ & $\ldots$ & $\ldots$ \\
\hline HoI & $\ldots$ & $\ldots$ & $\ldots$ & $1.1 \pm 0.5^{\mathrm{a}}$ & $\ldots$ & $\ldots$ & $<1.5^{\mathrm{d}}$ & $\ldots$ & $\ldots$ & $\ldots$ \\
\hline IC0342 & $\ldots$ & $430 \pm 110^{\mathrm{b}}$ & $\ldots$ & $860 \pm 160^{\mathrm{b}}$ & $\ldots$ & $\ldots$ & $1800 \pm 300^{\mathrm{b}}$ & $\ldots$ & 4.36 & $\ldots$ \\
\hline IC2574 & $\ldots$ & $8.3 \pm 1.3^{\mathrm{a}}$ & $\ldots$ & $10 \pm 1^{\mathrm{c}}$ & $\ldots$ & $\ldots$ & $19 \pm 8^{c}$ & $\ldots$ & 2.63 & $4.0_{0.5}^{0.9}$ \\
\hline M81DwB & $\ldots$ & $\ldots$ & $\ldots$ & $<0.46^{\mathrm{a}}$ & $\ldots$ & $\ldots$ & $\ldots$ & $\ldots$ & $\ldots$ & $\ldots$ \\
\hline NGC 0337 & $\ldots$ & $15 \pm 1^{\mathrm{a}}$ & $\ldots$ & $32 \pm 2^{\mathrm{a}}$ & $\ldots$ & $\ldots$ & $110 \pm 4^{\mathrm{d}}$ & $\ldots$ & 4.56 & $14.3_{1.3}^{1.8}$ \\
\hline NGC 0584 & $\ldots$ & $\ldots$ & $\ldots$ & $1.5 \pm 0.4^{\mathrm{a}}$ & $\ldots$ & $\ldots$ & $<1.5^{\mathrm{d}}$ & $\ldots$ & $\ldots$ & $\ldots$ \\
\hline NGC 0628 & $46 \pm 6^{\mathrm{e}}$ & $52 \pm 5^{\mathrm{a}}$ & $\ldots$ & $65 \pm 7^{\mathrm{a}}$ & $\ldots$ & $\ldots$ & $200 \pm 10^{\mathrm{a}}$ & $200 \pm 10^{\mathrm{f}}$ & 4.03 & $8.5_{1.3}^{1.5}$ \\
\hline NGC 0855 & $\ldots$ & $\ldots$ & $\ldots$ & $3.2 \pm 0.7^{\mathrm{a}}$ & $\ldots$ & $\ldots$ & $4.5^{\mathrm{d}}$ & $\ldots$ & $\ldots$ & $\ldots$ \\
\hline NGC 0925 & $38 \pm 6^{\mathrm{e}}$ & $\ldots$ & $\ldots$ & $\ldots$ & $\ldots$ & $\ldots$ & $90 \pm 10^{\mathrm{f}}$ & $\ldots$ & $\ldots$ & $\ldots$ \\
\hline NGC 1266 & $\ldots$ & $20 \pm 1^{\mathrm{a}}$ & $\ldots$ & $35.0 \pm 6.0^{\mathrm{a}}$ & $\ldots$ & $\ldots$ & $115 \pm 4^{\mathrm{d}}$ & $\cdots$ & 5.0 & $18.2_{4.2}^{4.7}$ \\
\hline NGC 1377 & $\cdots$ & $\ldots$ & $\cdots$ & $52.5 \pm 1.2^{\mathrm{a}}$ & $\cdots$ & $\ldots$ & $<1.5^{\mathrm{d}}$ & $\cdots$ & $\ldots$ & $\ldots$ \\
\hline NGC 1482 & $\ldots$ & $40.2 \pm 2.1^{\mathrm{a}}$ & $\ldots$ & $87.5 \pm 4.9^{\mathrm{a}}$ & $\ldots$ & $\ldots$ & $238 \pm 8^{\mathrm{d}}$ & $\ldots$ & 5.06 & $\ldots$ \\
\hline NGC 2146 & $224 \pm 6^{\mathrm{e}}$ & $\ldots$ & $472 \pm 25^{\mathrm{g}}$ & $439 \pm 21^{\mathrm{a}}$ & $\ldots$ & $\cdots$ & $1074 \pm 40^{\mathrm{d}}$ & $1100 \pm 10^{\mathrm{f}}$ & 5.59 & $27.3_{5.2}^{7.8}$ \\
\hline NGC 2798 & $\ldots$ & $23 \pm 1.5^{\mathrm{a}}$ & $\ldots$ & $33.8 \pm 2.5^{\mathrm{a}}$ & $\ldots$ & $\ldots$ & $82 \pm 3^{\mathrm{d}}$ & $\ldots$ & 4.83 & $19.1_{4.2}^{5.2}$ \\
\hline NGC 2841 & $14 \pm 10^{\mathrm{e}}$ & $\ldots$ & $34 \pm 11^{\mathrm{u}}$ & $38 \pm 4^{\mathrm{a}}$ & $\ldots$ & $45 \pm 9^{g}$ & $\ldots$ & $100 \pm 7^{f}$ & 4.30 & $15.0_{3.1}^{2.5}$ \\
\hline NGC 2976 & $21 \pm 3^{\mathrm{e}}$ & $\ldots$ & $\ldots$ & $39 \pm 3^{\mathrm{a}}$ & $\ldots$ & $\ldots$ & $125 \pm 10^{\mathrm{d}}$ & $\ldots$ & 3.18 & $6.7_{0.7}^{1.3}$ \\
\hline NGC 3049 & $\ldots$ & $\ldots$ & $\ldots$ & $4.8 \pm 0.4^{\mathrm{a}}$ & $\ldots$ & $8 \pm 4^{\mathrm{h}}$ & $12 \pm 2^{\mathrm{d}}$ & $\ldots$ & 3.73 & $8.8_{1.0}^{2.5}$ \\
\hline NGC 3077 & $13 \pm 1^{\mathrm{e}}$ & $\ldots$ & $\ldots$ & $23 \pm 1^{\mathrm{a}}$ & $\ldots$ & $\ldots$ & $30 \pm 2^{\mathrm{d}}$ & $\ldots$ & 2.88 & $\ldots$ \\
\hline NGC 3184 & $16 \pm 8^{\mathrm{e}}$ & $\ldots$ & $\ldots$ & $28 \pm 3^{\mathrm{a}}$ & $\ldots$ & $\cdots$ & $77 \pm 2^{w}$ & $80 \pm 5^{\mathrm{f}}$ & 4.06 & $8.7_{1.7}^{3.4}$ \\
\hline NGC 3190 & $15 \pm 7^{\mathrm{e}}$ & $\ldots$ & $\ldots$ & $13.5 \pm 0.5^{\mathrm{a}}$ & $\ldots$ & $22 \pm 3^{h}$ & $42 \pm 8^{t}$ & $\ldots$ & 4.18 & $13.5_{2.1}^{2.6}$ \\
\hline NGC 3198 & $<3^{\mathrm{e}}$ & $\ldots$ & $\ldots$ & $12 \pm 1^{\mathrm{a}}$ & $\ldots$ & $\ldots$ & $\ldots$ & $49 \pm 5^{\mathrm{f}}$ & $\ldots$ & $\ldots$ \\
\hline NGC 3265 & $\ldots$ & $3.5 \pm 0.5^{\mathrm{a}}$ & $\cdots$ & $5.7 \pm 0.6^{\mathrm{a}}$ & $\ldots$ & $\cdots$ & $10.1 \pm 0.9^{\mathrm{d}}$ & $\ldots$ & 3.72 & $8.2_{1.6}^{2.2}$ \\
\hline NGC 3351 & $14 \pm 2^{\mathrm{e}}$ & $\ldots$ & $\ldots$ & $\ldots$ & $\ldots$ & $\ldots$ & $43 \pm 10^{\mathrm{d}}$ & $\ldots$ & $\ldots$ & $\ldots$ \\
\hline NGC 3521 & $80 \pm 20^{\mathrm{e}}$ & $\ldots$ & $\ldots$ & $170 \pm 14^{\mathrm{i}}$ & $\ldots$ & $300 \pm 60^{\mathrm{j}}$ & $560 \pm 20^{\mathrm{a}}$ & $\ldots$ & 4.82 & $19.6_{2.2}^{2.3}$ \\
\hline NGC 3627 & $100 \pm 10^{\mathrm{e}}$ & $\ldots$ & $177 \pm 23^{\mathrm{u}}$ & $181 \pm 41^{\mathrm{b}}$ & $\ldots$ & $\ldots$ & $\ldots$ & $500 \pm 10^{\mathrm{f}}$ & 4.68 & $16.1_{4.5}^{5.4}$ \\
\hline NGC 3773 & $\ldots$ & $\ldots$ & $\ldots$ & $2.9 \pm 0.3^{\mathrm{a}}$ & $\ldots$ & $\ldots$ & $\ldots$ & $\ldots$ & $\ldots$ & $\ldots$ \\
\hline NGC 3938 & $15 \pm 4^{\mathrm{e}}$ & $\ldots$ & $\ldots$ & $26.3 \pm 1.5^{\mathrm{a}}$ & $\ldots$ & $\ldots$ & $\ldots$ & $80 \pm 5^{\mathrm{f}}$ & 4.04 & $9.1_{1.7}^{2.2}$ \\
\hline NGC 4236 & $9 \pm 1^{\mathrm{e}}$ & $\ldots$ & $\ldots$ & $23 \pm 3^{c}$ & $\ldots$ & $\ldots$ & $48 \pm 6^{c}$ & $\ldots$ & 3.07 & $\ldots$ \\
\hline NGC 4254 & $93 \pm 8^{\mathrm{e}}$ & $102 \pm 5^{\mathrm{k}}$ & $135 \pm 19^{\mathrm{u}}$ & $167 \pm 16^{\mathrm{k}}$ & $\ldots$ & $\ldots$ & $512 \pm 19^{\mathrm{k}}$ & $510 \pm 10^{\mathrm{f}}$ & 5.02 & $16.5_{3.0}^{2.1}$ \\
\hline NGC 4321 & $61 \pm 5^{\mathrm{e}}$ & $66 \pm 6^{\mathrm{b}}$ & $\ldots$ & $96 \pm 5^{1}$ & $\ldots$ & $\ldots$ & $\ldots$ & $310 \pm 10^{\mathrm{f}}$ & 4.79 & $13.3_{1.8}^{1.5}$ \\
\hline NGC 4536 & $39 \pm 3^{\mathrm{m}}$ & $42 \pm 4^{\mathrm{m}}$ & $\ldots$ & $80 \pm 2^{\mathrm{m}}$ & $\ldots$ & $\ldots$ & $205 \pm 20^{\mathrm{d}}$ & $\ldots$ & 4.69 & $17.3_{1.2}^{1.4}$ \\
\hline NGC 4559 & $18 \pm 11^{\mathrm{e}}$ & $\ldots$ & $31 \pm 11^{\mathrm{u}}$ & $38 \pm 3^{\mathrm{a}}$ & $\ldots$ & $\ldots$ & $100 \pm 4^{\mathrm{a}}$ & $110 \pm 10^{\mathrm{f}}$ & 3.68 & $9.3_{0.7}^{0.8}$ \\
\hline NGC 4569 & $30 \pm 6^{\mathrm{e}}$ & $36 \pm 10^{\mathrm{b}}$ & $\ldots$ & $57 \pm 20^{\mathrm{s}}$ & $\ldots$ & $\ldots$ & $\ldots$ & $170 \pm 10^{\mathrm{f}}$ & 4.13 & $11.7_{4.3}^{4.9}$ \\
\hline NGC 4579 & $82 \pm 4^{\mathrm{e}}$ & $60 \pm 10^{\mathrm{m}}$ & $57 \pm 17^{\mathrm{u}}$ & $99 \pm 10^{\mathrm{m}}$ & $\ldots$ & $\ldots$ & $167 \pm 25^{\mathrm{n}}$ & $\ldots$ & 4.84 & $\ldots$ \\
\hline NGC 4594 & $133 \pm 8^{\mathrm{e}}$ & $\ldots$ & $\ldots$ & $156 \pm 13^{\mathrm{i}}$ & $\ldots$ & $\ldots$ & $94 \pm 20^{\mathrm{d}}$ & $\ldots$ & $\ldots$ & $\ldots$ \\
\hline NGC 4625 & $\ldots$ & $\ldots$ & $\ldots$ & $3.1 \pm 0.3^{\mathrm{a}}$ & $\ldots$ & $\ldots$ & $7.1 \pm 0.2^{\mathrm{w}}$ & $\ldots$ & $\ldots$ & $\ldots$ \\
\hline NGC 4631 & $265 \pm 12^{\mathrm{e}}$ & $310 \pm 16^{\mathrm{b}}$ & $\ldots$ & $430 \pm 20^{\mathrm{b}}$ & $\ldots$ & $\ldots$ & $1122 \pm 50^{\mathrm{v}}$ & $\ldots$ & 4.69 & $24.7_{2.5}^{3.0}$ \\
\hline NGC 4725 & $\ldots$ & $19 \pm 1^{\mathrm{a}}$ & $\ldots$ & $30 \pm 2^{\mathrm{a}}$ & $\ldots$ & $\ldots$ & $92 \pm 3^{\mathrm{a}}$ & $100 \pm 10^{\mathrm{f}}$ & 4.11 & $10.2_{1.8}^{1.9}$ \\
\hline NGC 4736 & $90 \pm 18^{\mathrm{e}}$ & $\ldots$ & $111 \pm 10^{\mathrm{g}}$ & $125 \pm 10^{\mathrm{b}}$ & $\ldots$ & $\cdots$ & $295 \pm 5^{a}$ & $320 \pm 10^{\mathrm{f}}$ & 3.92 & $8.9_{1.9}^{1.5}$ \\
\hline NGC 4826 & $29 \pm 16^{\mathrm{e}}$ & $\ldots$ & $58 \pm 12^{\mathrm{u}}$ & $54 \pm 4^{\mathrm{a}}$ & $\ldots$ & $\ldots$ & $126 \pm 2^{\mathrm{a}}$ & $\ldots$ & 3.63 & $8.7_{1.7}^{2.3}$ \\
\hline NGC 5055 & $97 \pm 8^{\mathrm{e}}$ & $\ldots$ & $116 \pm 21^{\mathrm{u}}$ & $167 \pm 8^{a}$ & $254 \pm 51^{\mathrm{g}}$ & $260 \pm 20^{\mathrm{g}}$ & $460 \pm 5^{\mathrm{a}}$ & $450 \pm 10^{\mathrm{f}}$ & 4.49 & $14.1_{1.0}^{2.0}$ \\
\hline
\end{tabular}


Table 4

(Continued)

\begin{tabular}{|c|c|c|c|c|c|c|c|c|c|c|}
\hline $\begin{array}{l}\text { Galaxy } \\
\text { Name }\end{array}$ & $\begin{array}{c}S_{2.8 \mathrm{~cm}}^{10.7 \mathrm{cHz}} \\
(\mathrm{mJy})\end{array}$ & $\begin{array}{c}S_{3.6 \mathrm{GHz}}^{8.4 \mathrm{GH}} \\
(\mathrm{mJy})\end{array}$ & $\begin{array}{l}S_{6 \mathrm{~cm}}^{5 \mathrm{GHz}} \\
(\mathrm{mJy})\end{array}$ & $\begin{array}{c}S_{6.2 \mathrm{~cm}}^{4.8 \mathrm{GHz}} \\
(\mathrm{mJy})\end{array}$ & $\begin{array}{c}S_{8.1 \mathrm{~cm}}^{3.7 \mathrm{GHz}} \\
(\mathrm{mJy})\end{array}$ & $\begin{array}{c}S_{11.1 \mathrm{~cm}}^{2.7 \mathrm{cHz}} \\
(\mathrm{mJy})\end{array}$ & $\begin{array}{c}S_{20}^{1.4 \mathrm{~cm} z} \\
(\mathrm{mJy})\end{array}$ & $\begin{array}{c}S_{22 \mathrm{~cm}}^{1.36 \mathrm{GHz}} \\
(\mathrm{mJy})\end{array}$ & $\begin{array}{c}\log (\mathrm{MRC}) \\
{\left[L_{\odot}\right]}\end{array}$ & $\begin{array}{r}\mathrm{B} \\
(\mu \mathrm{G})\end{array}$ \\
\hline NGC 5457 & $152 \pm 62^{\mathrm{g}}$ & $\ldots$ & $\ldots$ & $310 \pm 20^{\mathrm{b}}$ & $\ldots$ & $442 \pm 30^{g}$ & $760 \pm 17^{\mathrm{a}}$ & $\ldots$ & 4.61 & $12.9_{1.9}^{1.2}$ \\
\hline NGC 5474 & $\ldots$ & $\ldots$ & $\ldots$ & $5.0 \pm 0.6^{\mathrm{a}}$ & $\ldots$ & $\ldots$ & $\ldots$ & $\ldots$ & $\ldots$ & $\ldots$ \\
\hline NGC 5713 & $41 \pm 3^{\mathrm{e}}$ & $31 \pm 1^{\mathrm{o}}$ & $\cdots$ & $58.8 \pm 2.7^{\mathrm{a}}$ & $\cdots$ & $\ldots$ & $158 \pm 6^{\mathrm{d}}$ & $\ldots$ & 4.89 & $16.4_{2.7}^{3.0}$ \\
\hline NGC 5866 & $\cdots$ & $9.1 \pm 0.6^{\mathrm{a}}$ & $13 \pm 6^{\mathrm{u}}$ & $12.1 \pm 0.8^{\mathrm{a}}$ & $\cdots$ & $\ldots$ & $22 \pm 1^{r}$ & $\ldots$ & 3.90 & $11.1_{3.2}^{6.0}$ \\
\hline NGC 6946 & $376 \pm 18^{b}$ & $422 \pm 65^{\mathrm{p}}$ & $\ldots$ & $660 \pm 50^{\mathrm{b}}$ & $\ldots$ & $794 \pm 75^{\mathrm{b}}$ & $1440 \pm 100^{\mathrm{p}}$ & $\ldots$ & 4.92 & $16.0_{3.0}^{2.4}$ \\
\hline NGC 7331 & $77 \pm 5^{\mathrm{e}}$ & $\cdots$ & $94 \pm 13^{\mathrm{u}}$ & $173.8 \pm 8.7^{\mathrm{a}}$ & $\ldots$ & $\cdots$ & $540 \pm 9^{a}$ & $\cdots$ & 5.00 & $23.6_{1.8}^{2.3}$ \\
\hline M51 & $235 \pm 32^{\mathrm{q}}$ & $306 \pm 26^{\mathrm{b}}$ & $\cdots$ & $420 \pm 80^{\mathrm{b}}$ & $\cdots$ & $780 \pm 50^{\mathrm{q}}$ & $1400 \pm 100^{\mathrm{x}}$ & $\cdots$ & 4.95 & $15.5_{3.4}^{3.5}$ \\
\hline
\end{tabular}

Notes. Upper limits at $20 \mathrm{~cm}$ refer to the $3 \sigma$ limit of the NVSS at these positions. The MRC luminosity is calculated using Equation (6).

a This work.

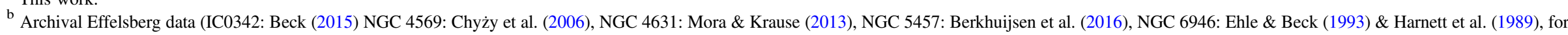

the rest see Stil et al. (2009)).

${ }^{c}$ Chyży et al. (2007a).

d Condon et al. (1998).

e Niklas et al. (1995).

${ }^{\mathrm{f}}$ Braun et al. (2007).

g Klein \& Emerson (1981).

a ${ }^{\mathrm{h}}$ Dressel \& Condon (1978).

${ }^{\mathrm{i}}$ Griffith et al. (1995) and Griffith et al. (1994).

${ }^{j}$ Parkes Catalogue, 1990, Australia Telescope National Facility.

${ }^{\mathrm{k}}$ Chyży et al. (2007b).

${ }^{1}$ Weżgowiec et al. (2012).

${ }^{\mathrm{m}}$ Vollmer et al. (2004).

${ }^{\mathrm{n}}$ Murphy et al. (2009).

${ }^{\circ}$ Schmitt et al. (2006).

$\mathrm{p}$ Tabatabaei et al. (2013c).

${ }^{\mathrm{q}}$ Klein et al. (1984).

${ }^{\mathrm{r}}$ Brown et al. (2011).

s average of measurements by Chyży et al. (2006) and Vollmer et al. (2004).

${ }^{\mathrm{t}}$ Gioia \& Fabbiano (1987).

u Sramek (1975).

${ }^{\mathrm{v}}$ White \& Becker (1992)

${ }^{\mathrm{w}}$ Condon et al. (2002).

${ }^{\mathrm{x}}$ Dumas et al. (2011).

(This table is available in machine-readable form.) 
image, before integration. The integrated radio flux densities can be uncertain in different ways via the calibration uncertainty, map fluctuations, and the baselevel uncertainty of the single-dish observations. The calibration error $\left(\delta_{\mathrm{cal}}\right)$ of the Effelsberg observations is $\simeq 5 \%$ at $3.6 \mathrm{~cm}$ and $6 \mathrm{~cm}$, and $\simeq 2 \%$ at $20 \mathrm{~cm}$ (the error in the absolute scale of the radio flux densities is similar, $\simeq 5$ percent, at different wavelengths, Baars et al. 1977). The error due to the map fluctuations is given by

$$
\delta_{\mathrm{rms}}=\sigma_{\mathrm{rms}} \sqrt{N_{\text {beam }}}=\sigma_{\mathrm{rms}} \frac{a}{\theta} \sqrt{\frac{N}{1.133}},
$$

where $\sigma_{\text {rms }}$ is the rms noise level, $N_{\text {beam }}$ the number of beams, $\theta$ the angular resolution, $N$ the number of pixels, and $a$ the pixel size. The error due to the baselevel uncertainty is $\delta_{\text {base }}=$ $\sigma_{0} N_{\text {beam }}$ with $\sigma_{0}$ the zero level uncertainty $\left(\sigma_{0}=0.2 \sigma_{\text {rms }}\right.$ for the Effelsberg measurements). The total error in the integrated flux densities is hence $\delta=\sqrt{\delta_{\text {cal }}^{2}+\delta_{\text {rms }}^{2}+\delta_{\text {base }}^{2}}$, which is $\simeq 7 \%$ at $3.6, \simeq 6 \%$ at $6 \mathrm{~cm}$, and $\simeq 4 \%$ at $20 \mathrm{~cm}$ averaged over the observed galaxy sample.

\subsection{Modeling the Radio SED}

The RC spectrum is often taken as the power law

$$
S_{\nu}=A \nu^{-\alpha},
$$

where $\alpha$ is the power-law index, $\nu$ is the frequency, and $A$ is a constant factor. However, at frequencies of $1<\nu<10 \mathrm{GHz}$, the $\mathrm{RC}$ emission is mainly due to two different mechanisms, the free-free emission from thermal electrons and the nonthermal emission from relativistic electrons. In terms of these mechanisms, and assuming the optically thin condition for the thermal emission, ${ }^{20}$ the RC spectrum can be expressed as

$$
S_{\nu}=S_{\nu}^{\mathrm{th}}+S_{\nu}^{\mathrm{nt}}=A_{1} \nu^{-0.1}+A_{2} \nu^{-\alpha_{\mathrm{nt}}},
$$

where $\alpha_{\mathrm{nt}}$ is the nonthermal spectral index and $A_{1}$ and $A_{2}$ are constant scaling factors. We note that, globally, $\alpha_{\text {nt }}$ represents the dominant energy loss mechanism experienced by the CRE population after injection from their sources in a galaxy over the $1-10 \mathrm{GHz}$ frequency range. To avoid dependencies on the units of the frequency space, Equation (3) can be written as

$$
S_{\nu}=A_{1}^{\prime}\left(\frac{\nu}{\nu_{0}}\right)^{-0.1}+A_{2} \nu_{0}^{-\alpha_{\mathrm{nt}}}\left(\frac{\nu}{\nu_{0}}\right)^{-\alpha_{\mathrm{nt}}},
$$

with $A_{1}^{\prime}=\nu_{0}^{-0.1} A_{1}$. The thermal fraction at the reference frequency $\nu_{0}$ is hence given by

$$
f_{\text {th }}\left(\nu_{0}\right) \equiv S_{\nu_{0}}^{\text {th }} / S_{\nu_{0}}=\frac{A_{1}^{\prime}}{S_{\nu_{0}}} .
$$

We used a Bayesian MCMC interface to fit the above model to the flux densities and derive the model parameters. This approach provides robust statistical constraints on the fit parameters because it is based on a wide library of models encompassing all plausible parameter combinations. Given an observed galaxy, the likelihood distribution of any physical parameter can be derived by evaluating how well

\footnotetext{
${ }^{20}$ The thermal term in this expression is equivalent to the Planck function for an optically thin ionized gas which is usually valid in the ISM and in starforming regions on $\geqslant$ sub-kiloparsec scales.
}

each model in the library accounts for the observed properties of the Galaxy. The underlying assumption is that the library of models is the distribution from which the data were randomly drawn. Thus, the prior distribution of models must be such that the entire observational space is reasonably well sampled, and that no a priori implausible corner of parameter space accounts for a large fraction of the models (e.g., da Cunha et al. 2008). We built a model library by generating random combinations of the parameters. To include all possible mechanisms of generation, acceleration, and cooling of CREs, we take $\alpha_{\text {nt }}$ to be uniformly distributed over the interval from 0 to 2.2 including injection with $\alpha_{\text {nt }} \sim 0.5-0.7$ (e.g., Longair 1994; Berkhuijsen 1986) to synchrotron and inverse Compton cooling with $\alpha_{\mathrm{nt}} \sim 1-1.2$. The normalization factors $A_{1}^{\prime}$ and $A_{2}$ are sampled uniformly in the wide ranges $-1<A_{1}^{\prime}<1$ and $-1<A_{2}<30$, leading to flux densities in Jy. The negative values are not physically motivated but are included to assess the robustness of the final results and particularly the necessity for the thermal term.

Using the emcee code (Foreman-Mackey et al. 2013), we obtained the range of probable values (posteriors) for each parameter. The median of the posterior probability distribution function (PDF) is then used as the reported result. The uncertainties were then taken as the median percentile $\pm 34 \%$ (or $16 \%, 84 \%$, equal-tailed interval). Figure 1 shows the posterior PDFs of $A_{1}^{\prime}, A_{2}$, and $\alpha_{\mathrm{nt}}$ for nine representative galaxies. The scatter plots between each posterior pair are also shown in the same figure. To have more constrained outputs, we applied this method to galaxies with $\geqslant 3$ data $^{\text {points. }}{ }^{21}$ Hence, the galaxies without enough detections/data points were excluded (DDO053, DDO154, DDO165, HoI, M81DwB, NGC 0584, NGC 0855, NGC 0925, NGC 1377, NGC 3198, NGC 3351, NGC 3773, NGC4625, and NGC5474).

\subsection{KINGFISHER Radio SED Parameters}

Figures 12-14 show the final modeled SEDs. Five galaxies, IC0342, NGC 1482, NGC 3077, NGC 4236, and NGC 4579, fit into the single-component model only. Fitting the doublecomponent model leads to negative thermal fractions in these galaxies that are not realistic and do not agree with other thermal-nonthermal decomposition methods (see the Appendix). Inconsistent radio flux densities collected from the archive, or the presence of variable radio-loud AGNs (as in the case of NGC 4579 hosting a LINER, e.g., Stauffer 1982) could cause this failure. It is also possible that $\alpha_{\mathrm{nt}}$ changes in the $1-10 \mathrm{GHz}$ frequency range for IC0342, NGC 1482, NGC 3077, and NGC 4236, due to the apparent curvature in their SED (Figures 12 and 13). However, this cannot be judged with only three data points available for these galaxies. Residuals between the thermal and nonthermal model and the observed fluxes are less than 20\% (modeled-observed/ observed) for most cases. Larger residuals are found at the high-frequency end for NGC 3190, NGC 4236, and NGC 5713. The galaxy NGC 4594 does not fit into either double- or single-component models because it shows an inverted spectrum. This galaxy is known to host a strong radio variable source (a LINER, see also Hummel et al. 1984). Hence, this galaxy was excluded from the rest of the analysis.

\footnotetext{
${ }^{21}$ We note that, unlike the $\chi^{2}$ method, the Bayesian method is not limited by the number of data points/degree of freedom because it looks for ranges of probable answers. Although a larger number of data points with smaller errors leads to more localized PDFs or smaller ranges of uncertainty.
} 

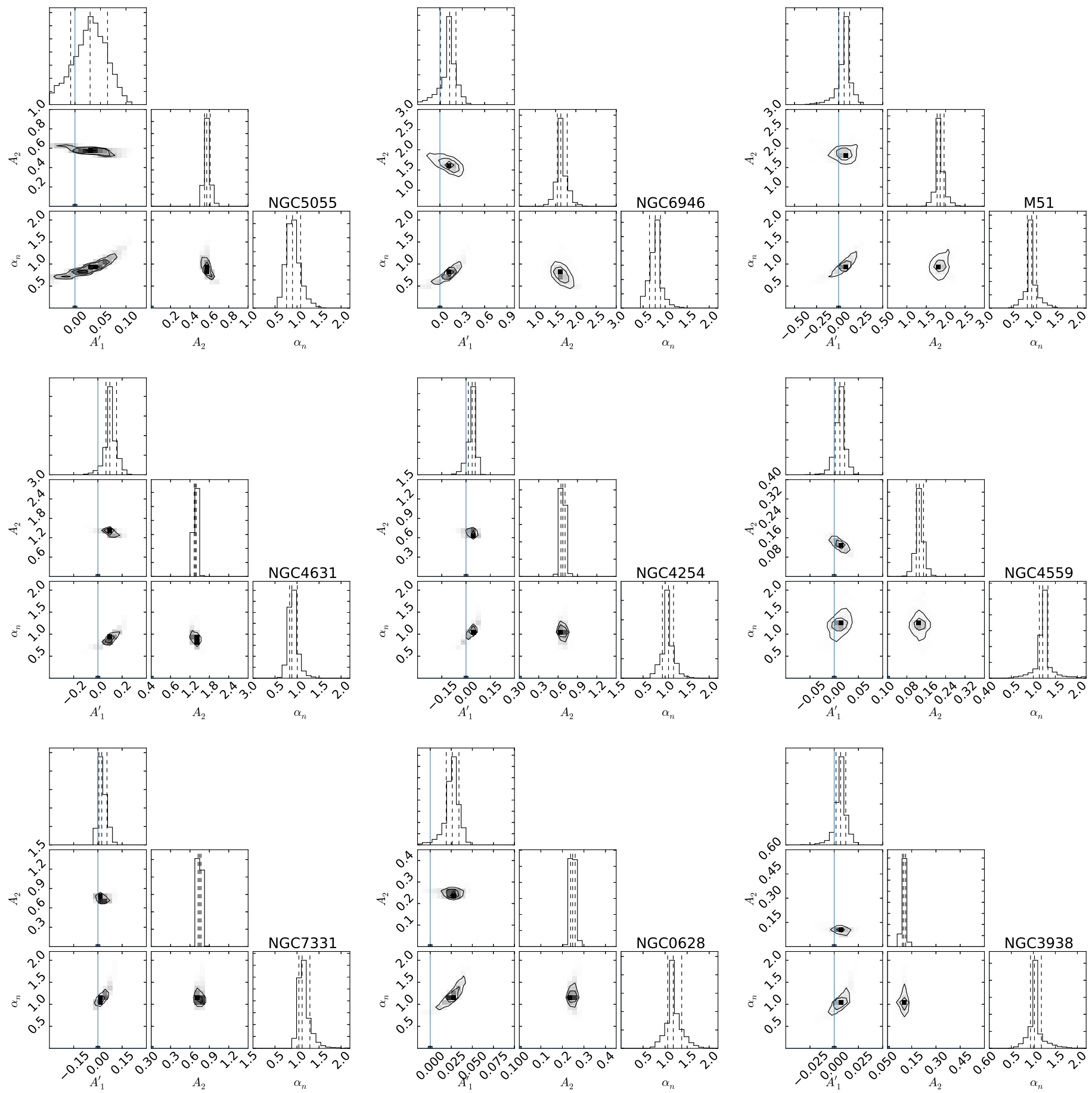

Figure 1. Bayesian corner plots for the parameters $A_{1}^{\prime}, A_{2}$, and $\alpha_{\mathrm{nt}}$ in Equation (5) showing the posterior probability distribution function (PDF) and their 0.16, 0.5, and 0.86 percentiles (dashed lines) for nine KINGFISHER galaxies. The uncertainty contours show that the posteriors have the highest probability to occur within the confidence intervals indicated.

The resulting $\alpha_{\mathrm{nt}}$, and the thermal fractions at $6 \mathrm{~cm}, f_{\mathrm{th}}(6 \mathrm{~cm})$, and at $20 \mathrm{~cm}, f_{\mathrm{th}}(20 \mathrm{~cm})$, together with their uncertainties are given in Table 5. Figure 2 illustrates the distribution of these parameters in the sample. The nonthermal spectral index changes between $0.57_{-0.16}^{+0.36}$ and $1.28_{-0.20}^{+0.32}$ with a mean of $\alpha_{\mathrm{nt}} \simeq 0.97$ (median of 0.99) and a standard deviation of 0.16 . The mean thermal fractions are $f_{\text {th }}(6 \mathrm{~cm})=(23 \pm 13) \%$ and $f_{\mathrm{th}}(20 \mathrm{~cm})=(10 \pm 9) \%$ over the entire sample and errors are the standard deviation. The dwarf irregular (Irr) galaxy IC 2574 shows the highest thermal fraction in the sample $\left(f_{\text {th }}(6 \mathrm{~cm}) \sim 55 \%, f_{\text {th }}(20 \mathrm{~cm}) \sim 35 \%\right)$. The relatively high thermal fraction in irregular galaxies was already known from previous studies in the Magellanic clouds (Loiseau et al. 1987; Jurusik et al. 2014). Plotting $\alpha_{\mathrm{nt}}$ against the thermal fractions given in Table 5, we see no obvious trend or correlation (Figure 3). Hence, the method did not introduce a correlation 
Table 5

Radio SED Parameters of the KINGFISHER Sample

\begin{tabular}{|c|c|c|c|c|}
\hline Galaxy & $\alpha_{\mathrm{nt}}$ & $f_{\mathrm{th}}(6 \mathrm{~cm})$ & $f_{\mathrm{th}}(20 \mathrm{~cm})$ & $\alpha$ \\
\hline $\mathrm{IC} 0342$ & $\ldots$ & $\ldots$ & $\ldots$ & $0.75_{0.11}^{0.14}$ \\
\hline IC2574 & $0.92_{0.07}^{0.21}$ & $0.55_{0.12}^{0.14}$ & $0.35_{0.06}^{0.08}$ & $0.50_{0.04}^{0.05}$ \\
\hline NGC 0337 & $1.13_{0.05}^{0.12}$ & $0.08_{0.03}^{0.09}$ & $0.03_{0.01}^{0.02}$ & $1.08_{0.03}^{0.04}$ \\
\hline NGC 0628 & $1.18_{0.13}^{0.17}$ & $0.44_{0.12}^{0.11}$ & $0.15_{0.04}^{0.04}$ & $0.84_{0.03}^{0.03}$ \\
\hline NGC 1266 & $1.03_{0.16}^{0.20}$ & $0.08_{0.20}^{0.15}$ & $0.03_{0.09}^{0.07}$ & $0.97_{0.03}^{0.03}$ \\
\hline NGC 1482 & $\cdots$ & $\ldots$ & $\ldots$ & $0.96_{0.03}^{0.03}$ \\
\hline NGC 2146 & $0.71_{0.13}^{0.20}$ & $0.02_{0.25}^{0.20}$ & $0.01_{0.12}^{0.10}$ & $0.68_{0.02}^{0.03}$ \\
\hline NGC 2798 & $0.73_{0.15}^{0.19}$ & $0.07_{0.18}^{0.10}$ & $0.03_{0.13}^{0.07}$ & $0.70_{0.03}^{0.03}$ \\
\hline NGC 2841 & $1.06_{0.19}^{0.14}$ & $0.22_{0.21}^{0.07}$ & $0.10_{0.11}^{0.04}$ & $0.81_{0.08}^{0.09}$ \\
\hline NGC 2976 & $1.13_{0.08}^{0.21}$ & $0.27_{0.14}^{0.20}$ & $0.09_{0.04}^{0.07}$ & $0.93_{0.07}^{0.07}$ \\
\hline NGC 3049 & $0.86_{0.06}^{0.24}$ & $0.31_{0.25}^{0.27}$ & $0.15_{0.13}^{0.14}$ & $0.75_{0.15}^{0.11}$ \\
\hline NGC 3077 & $\ldots$ & $\ldots$ & $\ldots$ & $0.40_{0.04}^{0.07}$ \\
\hline NGC 3184 & $1.06_{0.18}^{0.40}$ & $0.39_{0.20}^{0.25}$ & $0.15_{0.07}^{0.08}$ & $0.82_{0.12}^{0.17}$ \\
\hline NGC 3190 & $0.99_{0.15}^{0.19}$ & $0.18_{0.11}^{0.10}$ & $0.07_{0.05}^{0.4}$ & $0.89_{0.02}^{0.05}$ \\
\hline NGC 3265 & $0.85_{0.13}^{0.21}$ & $0.33_{0.07}^{0.10}$ & $0.19_{0.04}^{0.06}$ & $0.73_{0.02}^{0.05}$ \\
\hline NGC 3521 & $1.04_{0.08}^{0.09}$ & $0.15_{0.21}^{0.18}$ & $0.05_{0.06}^{0.04}$ & $0.95_{0.08}^{0.08}$ \\
\hline NGC 3627 & $0.89_{0.15}^{0.22}$ & $0.16_{0.24}^{0.20}$ & $0.06_{0.09}^{0.08}$ & $0.79_{0.02}^{0.03}$ \\
\hline NGC 3938 & $1.04_{0.16}^{0.23}$ & $0.28_{0.22}^{0.20}$ & $0.10_{0.09}^{0.08}$ & $0.87_{0.02}^{0.03}$ \\
\hline NGC 4236 & $\ldots$ & $\ldots$ & $\ldots$ & $0.76_{0.02}^{0.02}$ \\
\hline NGC 4254 & $1.03_{0.16}^{0.09}$ & $0.20_{0.14}^{0.09}$ & $0.07_{0.05}^{0.04}$ & $0.88_{0.03}^{0.03}$ \\
\hline NGC 4321 & $1.19_{0.15}^{0.12}$ & $0.43_{0.20}^{0.07}$ & $0.15_{0.10}^{0.02}$ & $0.84_{0.04}^{0.03}$ \\
\hline NGC 4536 & $0.91_{0.06}^{0.07}$ & $0.12_{0.04}^{0.06}$ & $0.04_{0.02}^{0.03}$ & $0.85_{0.05}^{0.05}$ \\
\hline NGC 4559 & $1.20_{0.03}^{0.05}$ & $0.31_{0.30}^{0.25}$ & $0.13_{0.12}^{0.10}$ & $0.92_{0.16}^{0.14}$ \\
\hline NGC 4569 & $1.28_{0.20}^{0.32}$ & $0.25_{0.18}^{0.15}$ & $0.10_{0.05}^{0.04}$ & $1.01_{0.09}^{0.08}$ \\
\hline NGC 4579 & $\ldots$ & $\ldots$ & $\ldots$ & $0.50_{0.03}^{0.03}$ \\
\hline NGC 4631 & $0.88_{0.08}^{0.10}$ & $0.23_{0.11}^{0.09}$ & $0.10_{0.05}^{0.04}$ & $0.73_{0.01}^{0.01}$ \\
\hline NGC 4725 & $1.10_{0.18}^{0.20}$ & $0.25_{0.15}^{0.13}$ & $0.08_{0.04}^{0.04}$ & $0.88_{0.01}^{0.02}$ \\
\hline NGC 4736 & $0.99_{0.19}^{0.15}$ & $0.25_{0.20}^{0.15}$ & $0.12_{0.05}^{0.04}$ & $0.73_{0.01}^{0.01}$ \\
\hline NGC 4826 & $0.87_{0.16}^{0.21}$ & $0.30_{0.27}^{0.25}$ & $0.15_{0.014}^{0.013}$ & $0.68_{0.04}^{0.05}$ \\
\hline NGC 5055 & $0.90_{0.05}^{0.12}$ & $0.17_{0.22}^{0.18}$ & $0.07_{0.08}^{0.06}$ & $0.78_{0.03}^{0.02}$ \\
\hline NGC 5457 & $0.97_{0.13}^{0.07}$ & $0.20_{0.16}^{0.13}$ & $0.08_{0.07}^{0.06}$ & $0.75_{0.03}^{0.04}$ \\
\hline NGC 5713 & $0.89_{0.14}^{0.16}$ & $0.04_{0.20}^{0.15}$ & $0.01_{0.10}^{0.08}$ & $0.87_{0.02}^{0.02}$ \\
\hline NGC 5866 & $0.57_{0.16}^{0.36}$ & $0.15_{0.15}^{0.20}$ & $0.10_{0.08}^{0.12}$ & $0.48_{0.04}^{0.04}$ \\
\hline NGC 6946 & $0.77_{0.13}^{0.10}$ & $0.24_{0.20}^{0.12}$ & $0.10_{0.08}^{0.05}$ & $0.67_{0.05}^{0.04}$ \\
\hline NGC 7331 & $1.10_{0.06}^{0.09}$ & $0.12_{0.13}^{0.15}$ & $0.04_{0.05}^{0.06}$ & $1.00_{0.01}^{0.02}$ \\
\hline M51 & $0.95_{0.10}^{0.09}$ & $0.15_{0.14}^{0.12}$ & $0.05_{0.05}^{0.04}$ & $0.86_{0.03}^{0.02}$ \\
\hline
\end{tabular}

between the final parameters, which, in principle, could occur due to simultaneous fitting and degeneracy.

In a separate run, we also determined the spectral index of the total continuum emission $\alpha$, following Equation (2), for the whole sample, which disregards the flattening by the thermal emission. A uniform prior was taken for $\alpha$ in the range of $0<\alpha<2.2$ and for the normalization factor $A$ in the range of $-1<A<30$. For the Galaxy sample, and in the $1.4-10.5 \mathrm{GHz}$ range, $\alpha$ changes from $0.40_{-0.04}^{+0.07}$ to $1.08_{-0.03}^{+0.04}$ with a mean value of $\alpha \simeq 0.79$ and a standard deviation of 0.15 (Table 5).

The average $\alpha$ and $\alpha_{\mathrm{nt}}$ are slightly higher than those reported by Israel \& van der Hulst (1983), Gioia et al. (1982), Klein \& Emerson (1981), and Niklas et al. (1997) because they included frequencies lower than $1 \mathrm{GHz}(\nu \sim 0.4-10.7 \mathrm{GHz})$, i.e., the SED flattening domain. It is important to note the wide range in the parameters. Most importantly, the synchrotron spectral index is not fixed in the sample (in agreement with Duric
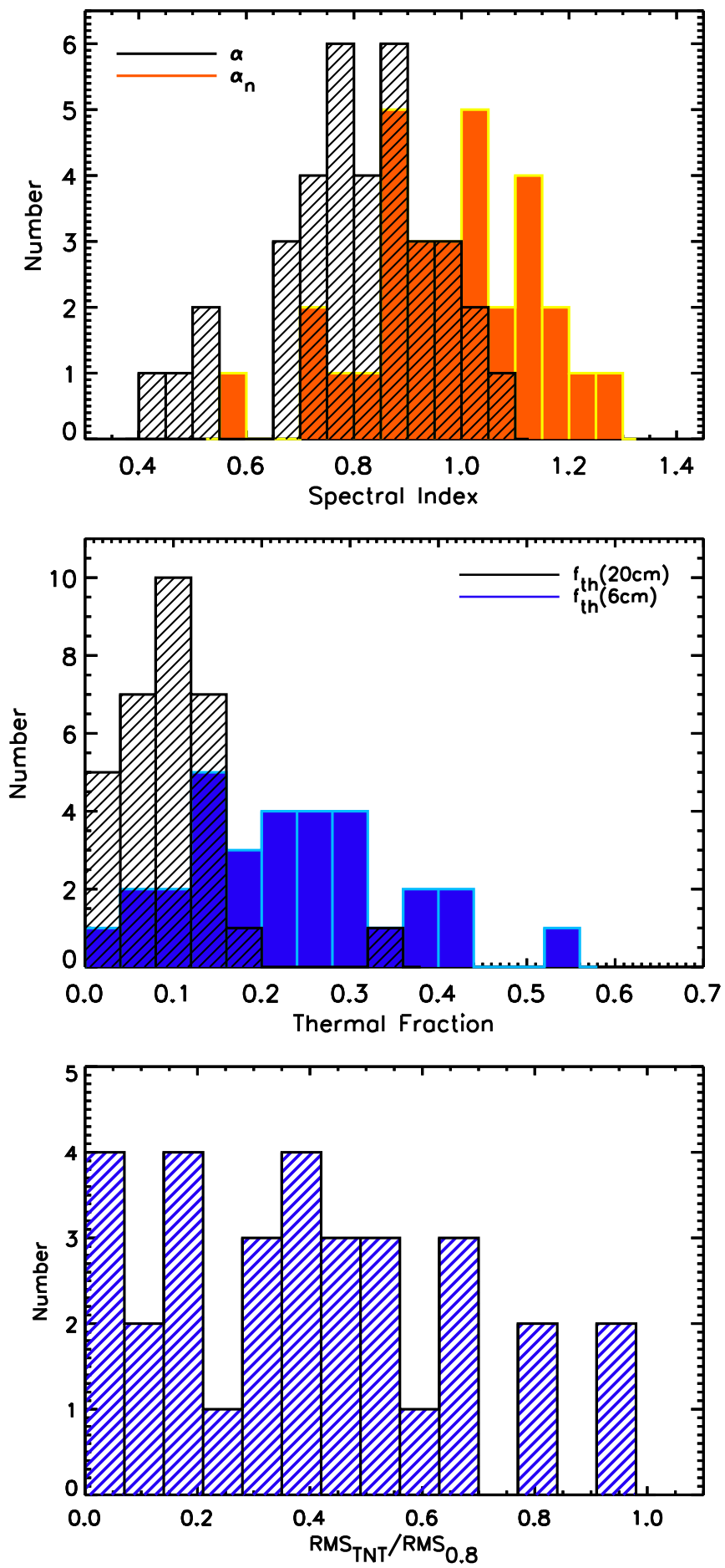

Figure 2. Top: histogram of the spectral index of the total radio continuum emission, $\alpha$, and its nonthermal component, $\alpha_{\mathrm{nt}}$, of the KINGFISHER sample. Middle: histogram of the thermal fractions at $6 \mathrm{~cm}$ and $20 \mathrm{~cm}$. Bottom: the root-mean-square deviation of the thermal + nonthermal model from the observation, $\mathrm{rms}_{\mathrm{TNT}}$, divided by the rms assuming a single power-law model with a fixed spectral index of $0.8\left(\mathrm{rms}_{0.8}\right)$. The first model leads to smaller deviations and hence it is more realistic (the median $\mathrm{rms}_{\mathrm{TNT}} / \mathrm{rms}_{0.8} \simeq 0.4$ ).

et al. 1988). We discuss the dependencies of $\alpha_{\text {nt }}$ on starformation properties in Section 7.1.

An almost common assumption about the radio SED is a single power-law model with a fixed spectral index of 0.8 . Figure 2 bottom panel, shows that this simple model leads, 


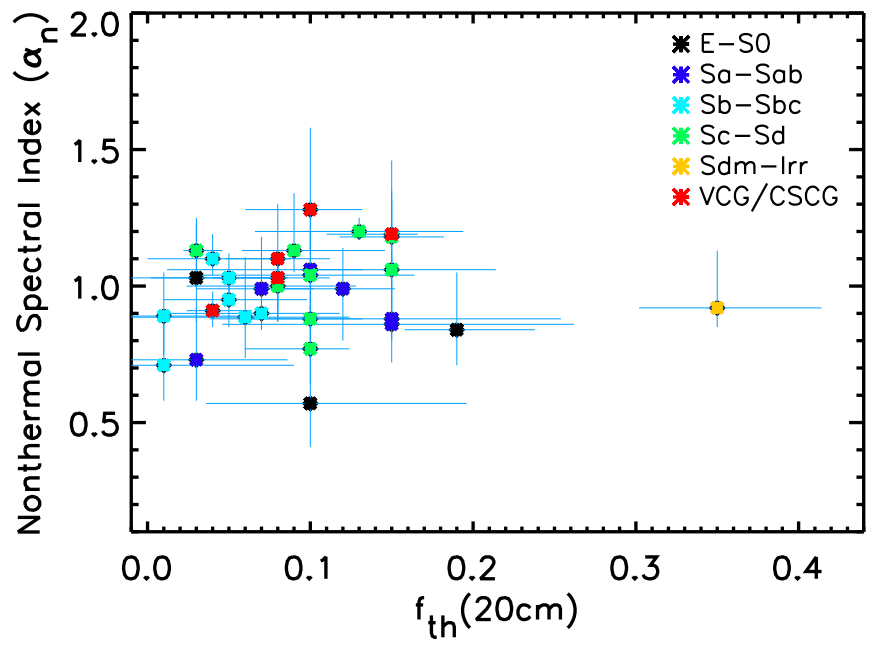

Figure 3. Nonthermal spectral index $\alpha_{\mathrm{nt}}$ against the thermal fraction at $20 \mathrm{~cm}$, $f_{\text {th }}(20 \mathrm{~cm})$, showing no correlation.

on average, to larger errors than the thermal + nonthermal model.

\section{Mid-RC Luminosity}

Integrating the SEDs over radio-frequency intervals is needed to study the total energy output of galaxies emitted in the radio. This would provide a quantitative way to study the energy balance between the radio and non-radio domains (e.g., the IR domain) of the electromagnetic radiation emitted from galaxies. The total energy budget of the RC emission in the $\mathrm{MRC}$, is given by

$$
\mathrm{MRC}=\int_{1.4}^{10.5} L_{\nu} d \nu
$$

with $L_{\nu}=4 \pi D^{2} S_{\nu}$ and using Equation (4) (Equation (2) for the few cases with the single power-law model as the only possibility). The integration was performed using Simpson's rule (see, e.g., Numerical Recipes by Press et al. 1992, 2nd edition, Section 4.2). The resulting MRC luminosities are listed in Table 4. The MRC bolometric luminosity varies over $\sim 3$ orders of magnitude in the sample, $4.3 \times 10^{2} L_{\odot}<$ MRC $<3.9 \times 10^{5} L_{\odot}$ (Figure 4$)$ with a mean luminosity of $4.8 \times 10^{4} L_{\odot}\left(\right.$ median of $\left.3.1 \times 10^{4} L_{\odot}\right)$. The thermal MRC luminosity,

$$
\mathrm{MRC}_{\mathrm{th}}=\int_{1.4}^{10.5} L_{\nu}^{\mathrm{th}} d \nu
$$

$\left(L_{\nu}^{\text {th }}=4 \pi D^{2} S_{\nu}^{\text {th }}\right.$ ) is about $5 \%-60 \%$ of the MRC, depending on the Galaxy. On average, the thermal emission provides about $23 \%$ of the total energy budget emitted at $1-10 \mathrm{GHz}$ in the sample.

To estimate the uncertainties in the MRC luminosities due to the uncertainties in the SED parameters $\alpha_{\text {nt }}$ and $f_{\text {th }}$, we first generated random data sets (100 mock data sets) assuming that they are uniformly distributed within their uncertainty intervals. Then the MRC integration (Equation (6)) was performed for each of these mock data sets. This leads to a distribution of 100 values for the MRC luminosity. We then took the $68 \%$ confidence interval $(1 \sigma)$ as the uncertainty value.

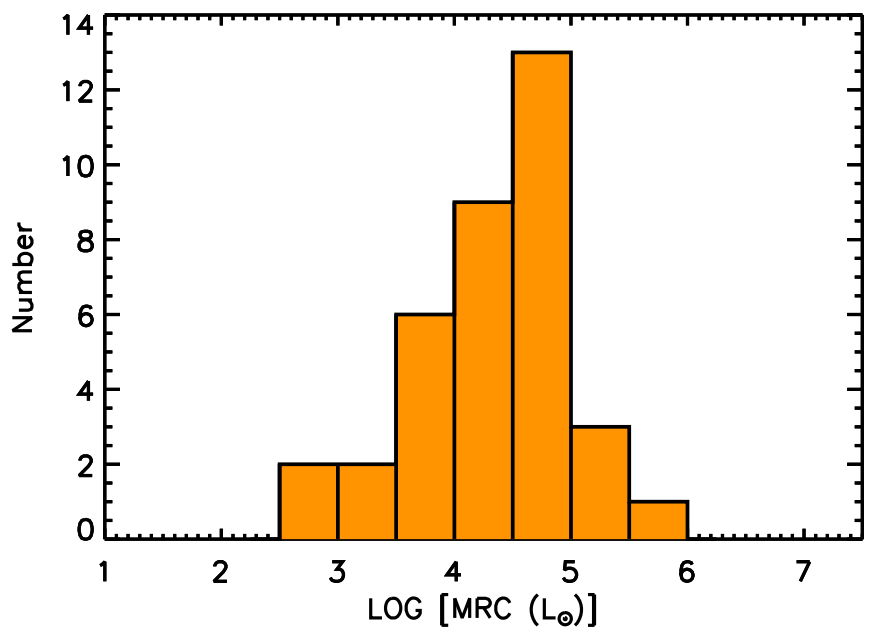

Figure 4. Distribution of the mid-radio continuum luminosity MRC of the galaxies.

Table 6

Relative Contribution of the Radio Bands in 1-12 GHz Bolometric Luminosity

\begin{tabular}{lcc}
\hline \hline Radio band & $S / S_{1-12 \mathrm{GHz}}$ & $S_{\text {th }} / S$ \\
\hline$L(1-2 \mathrm{GHz})$ & $24 \%$ & $10 \%$ \\
$S(2-4 \mathrm{GHz})$ & $26 \%$ & $17 \%$ \\
$C(4-8 \mathrm{GHz})$ & $30 \%$ & $27 \%$ \\
$X(8-12 \mathrm{GHz})$ & $20 \%$ & $38 \%$ \\
\hline
\end{tabular}

\subsection{Contribution of the Standard Bands to the MRC Radio Energy Budget}

Accounting for the Galaxy distances, the average radio SED is characterized and integrated over a slightly more extended frequency range of $1-12 \mathrm{GHz}$, which covers all four standard radio bands $L(1-2 \mathrm{GHz}), S(2-4 \mathrm{GHz}), C(4-8 \mathrm{GHz})$, and $X(8-12 \mathrm{GHz})$. To investigate the energetics and contributions of these standard bands to the $1-12 \mathrm{GHz}$ total energy budget, we determined the luminosity densities of the bands by integrating the average SED over the frequency width of the bands. Table 6 shows the band-to-total ratio of the luminosity densities as well as the thermal contribution at each band. The $C$ band centered at $6 \mathrm{~cm}$ provides the highest contribution in the total energy budget, though the band-to-band differences are not striking. Thermal sources provide $38 \%$ of the energy emitted in the $X$ band, highest among the bands as expected. Condon et al. (1991) modeled the radio spectrum of a sample of compact starbursts via

$$
\left\langle\frac{S_{\mathrm{nt}}}{S_{\mathrm{th}}}\right\rangle \sim 10\left(\frac{\nu}{1 \mathrm{GHz}}\right)^{0.1-\alpha_{\mathrm{nt}}} .
$$

Taking the same $\alpha_{\mathrm{nt}}$ as that of the average $\operatorname{SED}\left(\alpha_{\mathrm{nt}} \simeq 1\right)$, this model leads to $13 \%, 21 \%, 33 \%$, and $44 \%$ thermal fraction at the central frequencies of the $L, S, C$, and $X$ bands, respectively, which are slightly higher than the bolometric measurements in Table 6. Instead, the relation,

$$
\left\langle\frac{S_{\mathrm{nt}}}{S_{\mathrm{th}}}\right\rangle \sim 13\left(\frac{\nu}{1 \mathrm{GHz}}\right)^{0.1-\alpha_{\mathrm{nt}}},
$$

reproduces the thermal fractions at mid-radio frequencies with a higher precision for the average SED in the sample. 


\section{Radio-based Calibrations}

Measuring the rate at which massive stars form in galaxies is key to understand the formation and evolution of galaxies. Various lines and continuum emission data have been used so far as SFR diagnostics, each with advantages and shortcomings (for a review, see Kennicutt \& Evans 2012). The most frequently used tracers, $\mathrm{H} \alpha$ and UV (rest frame 125-250 nm) emission, are directly related to the massive star-formation process, but they could be obscured or attenuated by interstellar dust. This has motivated the use of hybrid star-formation tracers combining two or more different tracers including the IR emission to correct for the dust attenuation. The use of the IR emission itself as an SFR tracer is shadowed by a contribution from other sources/ mechanisms irrelevant to massive star formation such as interstellar dust heating by solar-mass stars (e.g., Xu 1990; Calzetti et al. 2010) and emission from the atmosphere of carbon stars (mainly in mid-IR, e.g., Verley et al. 2009; Tabatabaei \& Berkhuijsen 2010; Lu et al. 2014). The RC emission is an ideal SFR tracer because (a) it is not attenuated by dust, (b) it emerges from different phases of massive star formation from young stellar objects to H II regions and supernova remnants (SNRs), and (c) no other tracer is needed to be combined with. Even the diffuse emission, which is mainly nonthermal (e.g., Tabatabaei et al. 2007), traces massive stars in normal star-forming galaxies $^{22}$ but those occurred in the past: the CRE lifetime is $t_{\mathrm{syn}} \simeq 1.06 \times 10^{9} \mathrm{yr}\left(\frac{B}{\mu \mathrm{G}}\right)^{-1.5}\left(\frac{\nu}{\mathrm{GHz}}\right)^{-0.5} \sim 10 \mathrm{Myr}$ at $6 \mathrm{~cm}$ $(\nu=4.85 \mathrm{GHz}$ ) where $B=13.5 \mu \mathrm{G}$ (see Section 6). Hence, the radio SFRs must provide a more precise measure of the rate of massive star formation in a galaxy than the common nonradio SFRs.

As follows, we calibrate the SFR, globally, using the monochromatic radio luminosities at 6 and $20 \mathrm{~cm}$. The radio SFR tracers are further compared with the standard non-radio tracers. We also present an SFR calibration relation using the bolometric MRC luminosity. Moreover, we construct an MRC calibration relation using the monochromatic radio luminosities at 6 and $20 \mathrm{~cm}$.

\subsection{Comparison of Radio SFRs with Standard SFR Diagnostics}

Taking advantage of the thermal and nonthermal emission separated through the SED analysis, we can now derive the radio SFR calibration relations directly and independently from the IR SFR relations (i.e., the radio-IR correlation, e.g., Condon et al. 2002). We further compare the radio and the commonly used SFR tracers, the $24 \mu \mathrm{m}, \mathrm{H} \alpha$, and FUV emission. A good correlation between those SFR tracers is the first requirement to calibrate the non-radio SFRs with the radio SFRs, particularly the thermal radio SFR as an ideal starformation diagnostic (Murphy et al. 2011).

Assuming a solar metallicity and continuous star formation, and using a Kroupa IMF, Murphy et al. (2011) obtained a

\footnotetext{
${ }^{22}$ The diffuse synchrotron emission in starburst galaxies is likely dominated by secondary CREs produced in their ISM dense gas (e.g., Lacki \& Beck 2013).
}

general calibration relation for the thermal radio emission:

$$
\begin{aligned}
\left(\frac{\mathrm{SFR}_{\nu}^{\mathrm{th}}}{M_{\odot} \mathrm{yr}^{-1}}\right)= & 4.6 \times 10^{-28}\left(\frac{T_{e}}{10^{4} \mathrm{~K}}\right)^{-0.45} \\
& \times\left(\frac{\nu}{\mathrm{GHz}}\right)^{0.1}\left(\frac{L_{\nu}^{\mathrm{th}}}{\mathrm{erg} \mathrm{s}^{-1} \mathrm{~Hz}^{-1}}\right),
\end{aligned}
$$

where $T_{e}$ is the electron temperature and $L_{\nu}^{\text {th }}$ is the thermal radio luminosity. At $6 \mathrm{~cm}$ and for $T_{e}=10^{4} \mathrm{~K}$, this becomes

$$
\left(\frac{\mathrm{SFR}_{6 \mathrm{~cm}}^{\mathrm{th}}}{M_{\odot} \mathrm{yr}^{-1}}\right)=1.11 \times 10^{-37}\left(\frac{\nu L_{\nu}^{\mathrm{th}}(6 \mathrm{~cm})}{\mathrm{erg} \mathrm{s}^{-1}}\right),
$$

We note that the electron temperature could exceed the typical value of $T_{e}=10^{4} \mathrm{~K}$ in low-metallicity dwarf galaxies. A mean temperature of $T_{e}=14,000 \mathrm{~K}$ has been found to be more representative in these objects (Nicholls et al. 2014), leading to a $14 \%$ decrease in the above calibration factor. Similarly, the thermal radio SFR at $20 \mathrm{~cm}$ is

$$
\left(\frac{\mathrm{SFR}_{20 \mathrm{~cm}}^{\mathrm{th}}}{M_{\odot} \mathrm{yr}^{-1}}\right)=3.29 \times 10^{-37}\left(\frac{\nu L_{\nu}^{\mathrm{th}}(20 \mathrm{~cm})}{\mathrm{erg} \mathrm{s}^{-1}}\right) .
$$

Calibrating between the supernova rate and the SFR using the output of Starburst99, and using the empirical relations between supernova rate and nonthermal spectral luminosity of the Milky Way (Tammann et al. 1982; Condon \& Yin 1990), Murphy et al. (2011) found the following relation for the nonthermal synchrotron emission,

$$
\begin{aligned}
\left(\frac{\mathrm{SFR}_{\nu}^{\mathrm{nt}}}{M_{\odot} \mathrm{yr}^{-1}}\right)= & 6.64 \times 10^{-29}\left(\frac{\nu}{\mathrm{GHz}}\right)^{\alpha_{\mathrm{nt}}} \\
& \times\left(\frac{L_{\nu}^{\mathrm{nt}}}{\operatorname{erg~s}^{-1} \mathrm{~Hz}^{-1}}\right)
\end{aligned}
$$

At $6 \mathrm{~cm}$, one obtains

$$
\begin{aligned}
\left(\frac{\mathrm{SFR}_{6 \mathrm{~cm}}^{\mathrm{nt}}}{M_{\odot} \mathrm{yr}^{-1}}\right)= & 1.37 \times 10^{-38}(4.85)^{\alpha_{\mathrm{nt}}} \\
& \times\left(\frac{\nu L_{\nu}^{\mathrm{nt}}(6 \mathrm{~cm})}{\mathrm{erg} \mathrm{s}^{-1}}\right),
\end{aligned}
$$

and at $20 \mathrm{~cm}$,

$$
\begin{aligned}
\left(\frac{\mathrm{SFR}_{20 \mathrm{~cm}}^{\mathrm{nt}}}{M_{\odot} \mathrm{yr}^{-1}}\right)= & 4.58 \times 10^{-38}(1.45)^{\alpha_{\mathrm{nt}}} \\
& \times\left(\frac{\nu L_{\nu}^{\mathrm{nt}}(20 \mathrm{~cm})}{\mathrm{erg} \mathrm{s}^{-1}}\right) .
\end{aligned}
$$

The $\alpha_{\text {nt }}$ determined in Section 3.1 (see Table 5) was used in the above relations (Equations (13) and (14)) to calculate the nonthermal radio SFRs at $6 \mathrm{~cm}$ and $20 \mathrm{~cm}$.

Because the total RC emission is a combination of the thermal and nonthermal emission, Equations (9) and (12) lead to the following general expression for the SFR based on the 
RC (Murphy et al. 2011):

$$
\begin{aligned}
\left(\frac{\mathrm{SFR}_{\nu}^{\mathrm{RC}}}{M_{\odot} \mathrm{yr}^{-1}}\right)= & 10^{-27}\left[2.18\left(\frac{T_{e}}{10^{4} \mathrm{~K}}\right)^{0.45}\right. \\
& \left.\times\left(\frac{\nu}{\mathrm{GHz}}\right)^{-0.1}+15.1\left(\frac{\nu}{\mathrm{GHz}}\right)^{-\alpha_{\mathrm{nt}}}\right]^{-1} \\
& \times\left(\frac{L_{\nu}}{\mathrm{erg} \mathrm{s}^{-1} \mathrm{~Hz}^{-1}}\right) .
\end{aligned}
$$

For instance, the case of $T_{e}=10^{4} \mathrm{~K}$ and $\alpha_{\mathrm{nt}}=1$ leads to the following SFR calibration relations:

$$
\left(\frac{\mathrm{SFR}_{6 \mathrm{~cm}}^{\mathrm{RC}}}{M_{\odot} \mathrm{yr}^{-1}}\right)=4.1 \times 10^{-38}\left(\frac{\nu L_{6 \mathrm{~cm}}}{\mathrm{erg} \mathrm{s}^{-1}}\right)
$$

and

$$
\left(\frac{\mathrm{SFR}_{20 \mathrm{~cm}}^{\mathrm{RC}}}{M_{\odot} \mathrm{yr}^{-1}}\right)=5.5 \times 10^{-38}\left(\frac{\nu L_{20 \mathrm{~cm}}}{\mathrm{erg} \mathrm{s}^{-1}}\right) .
$$

Because non-radio extinction-corrected SFR tracers, the $24 \mu \mathrm{m}$ emission as well as the hybrid diagnostics $\mathrm{H} \alpha+24 \mu \mathrm{m}$ and FUV $+24 \mu \mathrm{m}$ were used. The hybrid diagnostics could be expressed as the $\mathrm{H} \alpha$ and FUV emission corrected for extinction. The observed $\mathrm{H} \alpha$ luminosity is corrected following Kennicutt et al. (2009):

$$
L_{\mathrm{H} \alpha_{\text {corr }}}=L_{\mathrm{H} \alpha_{\mathrm{obs}}}+0.02 L_{24 \mu \mathrm{m}} .
$$

We corrected the FUV emission for obscursion by dust using the Hao et al. (2011) calibration relation given for galaxy luminosities:

$$
L_{\mathrm{FUV}_{\mathrm{corr}}}=L_{\mathrm{FUV}_{\mathrm{obs}}}+3.89 L_{24 \mu \mathrm{m}} .
$$

We note that, in this relation, the calibration factor of the $24 \mu \mathrm{m}$ term could change galaxy-by-galaxy depending on their stellar population and their contribution in the interstellar radiation field as shown by Boquien et al. (2016) for few KINGFISH galaxies.

The SFR can be estimated using the corrected $\mathrm{H} \alpha$ luminosity,

$$
\left(\frac{\mathrm{SFR}_{\mathrm{H} \alpha_{\text {corr }}}}{M_{\odot} \mathrm{yr}^{-1}}\right)=5.37 \times 10^{-42}\left(\frac{L_{\mathrm{H} \alpha_{\text {corr }}}}{\mathrm{erg} \mathrm{s}^{-1}}\right),
$$

which is a measure of the current star-formation activity ( $\lesssim 10$ Myr; Murphy et al. 2011).

The FUV emission traces a wider range of stellar ages and is sensitive to recent $(\lesssim 100 \mathrm{Myr})$ star-formation activity (Kenni-

\begin{tabular}{|c|c|c|c|c|c|}
\hline$X$ & $Y$ & $b$ & $a$ & $r$ & $\sigma$ \\
\hline \multicolumn{6}{|l|}{ (I) } \\
\hline $\mathrm{SFR}_{6 \mathrm{~cm}}^{\mathrm{th}}$ & $\mathrm{SFR}_{\mathrm{H} \alpha}$ & $1.13 \pm 0.13$ & $-0.03 \pm 0.06$ & 0.77 & 0.32 \\
\hline $\mathrm{SFR}_{6 \mathrm{~cm}}^{\mathrm{nt}}$ & $\mathrm{SFR}_{\mathrm{H} \alpha}$ & $0.89 \pm 0.08$ & $-0.33 \pm 0.07$ & 0.75 & 0.32 \\
\hline $\mathrm{SFR}_{6 \mathrm{~cm}}^{\mathrm{RC}}$ & $\mathrm{SFR}_{\mathrm{H} \alpha}$ & $0.94 \pm 0.08$ & $-0.27 \pm 0.06$ & 0.78 & 0.32 \\
\hline $\mathrm{SFR}_{20 \mathrm{~cm}}^{\text {th }}$ & $\mathrm{SFR}_{\mathrm{H} \alpha}$ & $1.12 \pm 0.13$ & $0.01 \pm 0.05$ & 0.74 & 0.34 \\
\hline $\mathrm{SFR}_{20 \mathrm{~cm}}^{\mathrm{nt}}$ & $\mathrm{SFR}_{\mathrm{H} \alpha}$ & $0.88 \pm 0.08$ & $-0.32 \pm 0.07$ & 0.76 & 0.34 \\
\hline $\mathrm{SFR}_{20 \mathrm{~cm}}^{\mathrm{RC}}$ & $\mathrm{SFR}_{\mathrm{H} \alpha}$ & $0.90 \pm 0.08$ & $-0.29 \pm 0.06$ & 0.77 & 0.33 \\
\hline $\mathrm{SFR}_{6 \mathrm{~cm}}^{\mathrm{th}}$ & SFR $_{\text {FUV }}$ & $1.11 \pm 0.11$ & $0.03 \pm 0.03$ & 0.86 & 0.27 \\
\hline $\mathrm{SFR}_{6 \mathrm{~cm}}^{\mathrm{nt}}$ & $\mathrm{SFR}_{\mathrm{FUV}}$ & $0.80 \pm 0.08$ & $-0.29 \pm 0.06$ & 0.89 & 0.24 \\
\hline $\mathrm{SFR}_{6 \mathrm{~cm}}^{\mathrm{RC}}$ & SFR $_{\mathrm{FUV}}$ & $0.86 \pm 0.07$ & $-0.23 \pm 0.05$ & 0.91 & 0.22 \\
\hline $\mathrm{SFR}_{20 \mathrm{~cm}}^{\mathrm{th}}$ & $\mathrm{SFR}_{\mathrm{FUV}}$ & $1.15 \pm 0.12$ & $0.07 \pm 0.03$ & 0.83 & 0.30 \\
\hline $\mathrm{SFR}_{20 \mathrm{~cm}}^{\mathrm{nt}}$ & $\mathrm{SFR}_{\mathrm{FUV}}$ & $0.78 \pm 0.07$ & $-0.25 \pm 0.05$ & 0.89 & 0.24 \\
\hline $\mathrm{SFR}_{20 \mathrm{~cm}}^{\mathrm{RC}}$ & SFR $_{\text {FUV }}$ & $0.81 \pm 0.07$ & $-0.23 \pm 0.06$ & 0.90 & 0.23 \\
\hline $\mathrm{SFR}_{6 \mathrm{~cm}}^{\mathrm{th}}$ & $\mathrm{SFR}_{24 \mu \mathrm{m}}$ & $1.07 \pm 0.10$ & $-0.04 \pm 0.04$ & 0.84 & 0.28 \\
\hline $\mathrm{SFR}_{6 \mathrm{~cm}}^{\mathrm{nt}}$ & $\mathrm{SFR}_{24 \mu \mathrm{m}}$ & $0.76 \pm 0.04$ & $-0.33 \pm 0.04$ & 0.94 & 0.17 \\
\hline $\mathrm{SFR}_{6 \mathrm{~cm}}^{\mathrm{RC}}$ & $\mathrm{SFR}_{24 \mu \mathrm{m}}$ & $0.81 \pm 0.04$ & $-0.28 \pm 0.03$ & 0.95 & 0.15 \\
\hline $\mathrm{SFR}_{20 \mathrm{~cm}}^{\text {th }}$ & $\mathrm{SFR}_{24 \mu \mathrm{m}}$ & $1.08 \pm 0.10$ & $0.01 \pm 0.04$ & 0.82 & 0.30 \\
\hline $\mathrm{SFR}_{20 \mathrm{~cm}}^{\mathrm{nt}}$ & $\mathrm{SFR}_{24 \mu \mathrm{m}}$ & $0.74 \pm 0.04$ & $-0.31 \pm 0.04$ & 0.95 & 0.16 \\
\hline $\begin{array}{l}\mathrm{SFR}_{20 \mathrm{~cm}}^{\mathrm{RC}} \\
\text { (II) }\end{array}$ & $\mathrm{SFR}_{24 \mu \mathrm{m}}$ & $0.77 \pm 0.04$ & $-0.29 \pm 0.04$ & 0.95 & 0.16 \\
\hline $\mathrm{SFR}_{6 \mathrm{~cm}}^{\mathrm{RC}}$ & $\mathrm{SFR}_{\mathrm{H} \alpha}$ & $0.80 \pm 0.07$ & $-0.17 \pm 0.05$ & 0.79 & 0.33 \\
\hline $\mathrm{SFR}_{20 \mathrm{~cm}}^{\mathrm{RC}}$ & $\mathrm{SFR}_{\mathrm{H} \alpha}$ & $0.79 \pm 0.06$ & $-0.22 \pm 0.05$ & 0.81 & 0.31 \\
\hline $\mathrm{SFR}_{\mathrm{MRC}}$ & $\mathrm{SFR}_{\mathrm{H} \alpha}$ & $1.05 \pm 0.07$ & $0.11 \pm 0.02$ & 0.85 & 0.28 \\
\hline $\mathrm{SFR}_{6 \mathrm{~cm}}^{\mathrm{RC}}$ & $\mathrm{SFR}_{\mathrm{FUV}}$ & $0.82 \pm 0.08$ & $-0.19 \pm 0.06$ & 0.88 & 0.26 \\
\hline $\mathrm{SFR}_{20 \mathrm{~cm}}^{\mathrm{RC}}$ & $\mathrm{SFR}_{\mathrm{FUV}}$ & $0.78 \pm 0.07$ & $-0.22 \pm 0.05$ & 0.90 & 0.24 \\
\hline $\mathrm{SFR}_{\mathrm{MRC}}$ & SFR $_{\text {FUV }}$ & $1.03 \pm 0.08$ & $0.10 \pm 0.02$ & 0.91 & 0.23 \\
\hline $\mathrm{SFR}_{6 \mathrm{~cm}}^{\mathrm{RC}}$ & $\mathrm{SFR}_{24 \mu \mathrm{m}}$ & $0.76 \pm 0.05$ & $-0.23 \pm 0.04$ & 0.93 & 0.20 \\
\hline $\mathrm{SFR}_{20 \mathrm{~cm}}^{\mathrm{RC}}$ & $\mathrm{SFR}_{24 \mu \mathrm{m}}$ & $0.75 \pm 0.04$ & $-0.26 \pm 0.03$ & 0.95 & 0.18 \\
\hline $\mathrm{SFR}_{\mathrm{MRC}}$ & $\mathrm{SFR}_{24 \mu \mathrm{m}}$ & $1.00 \pm 0.04$ & $0.04 \pm 0.02$ & 0.96 & 0.15 \\
\hline
\end{tabular}
cutt 1998; Calzetti et al. 2005). As in Murphy et al. (2011), we derived the SFR based on the corrected FUV luminosity using

$$
\left(\frac{\mathrm{SFR}_{\mathrm{FUV}_{\mathrm{corr}}}}{M_{\odot} \mathrm{yr}^{-1}}\right)=4.42 \times 10^{-44}\left(\frac{L_{\mathrm{FUV}_{\text {corr }}}}{\mathrm{erg} \mathrm{s}^{-1}}\right)
$$

Table 7

SFR Calibrations Using Radio Continuum

Note. The linear fits in logarithmic scales $(\log Y=b \log X+a)$ obtained using the bisector least square fit (Isobe et al. 1990) with $\sigma$ representing the scatter around the fit for (I) the galaxies with both the thermal and nonthermal components (Figure 5) and (II) the entire sample (Figure 6).

The mid-IR emission at $24 \mu \mathrm{m}$ has been widely used as an SFR tracer as well (e.g., Wu et al. 2005; Calzetti et al. 2007; Rieke et al. 2009). This emission also traces the star-formation activity over $\lesssim 100 \mathrm{Myr}$ (Kennicutt \& Evans 2012). We used the relation given by Relaño et al. (2007), which was calibrated for a wide range of the $24 \mu \mathrm{m}$ luminosities $\left(10^{38} \mathrm{erg} \mathrm{s}^{-1}<L_{24 \mu \mathrm{m}}<3 \times 10^{44} \mathrm{erg} \mathrm{s}^{-1}\right)$ using a Kroupa IMF:

$$
\left(\frac{\mathrm{SFR}_{24 \mu \mathrm{m}}}{M_{\odot} \mathrm{yr}^{-1}}\right)=5.58 \times 10^{-36}\left(\frac{\nu L_{24 \mu \mathrm{m}}}{\mathrm{erg} \mathrm{s}^{-1}}\right)^{0.826} .
$$

The monochromatic RC emission at 6 and $20 \mathrm{~cm}$ are well correlated with the above tracers. The Pearson correlation coefficients are $r>0.7$ between the radio and the non-radio SFRs (Table 7). The relations with the thermal radio SFRs agree within the errors and are closer to linearity compared to those with the nonthermal radio SFRs, though their scatter $\sigma$ can be larger (in the cases of $\mathrm{SFR}_{\mathrm{FUV}}$ and $\mathrm{SFR}_{24 \mu \mathrm{m}}$ ). This is seen better in Figure 5 showing the non-radio SFRs versus the 

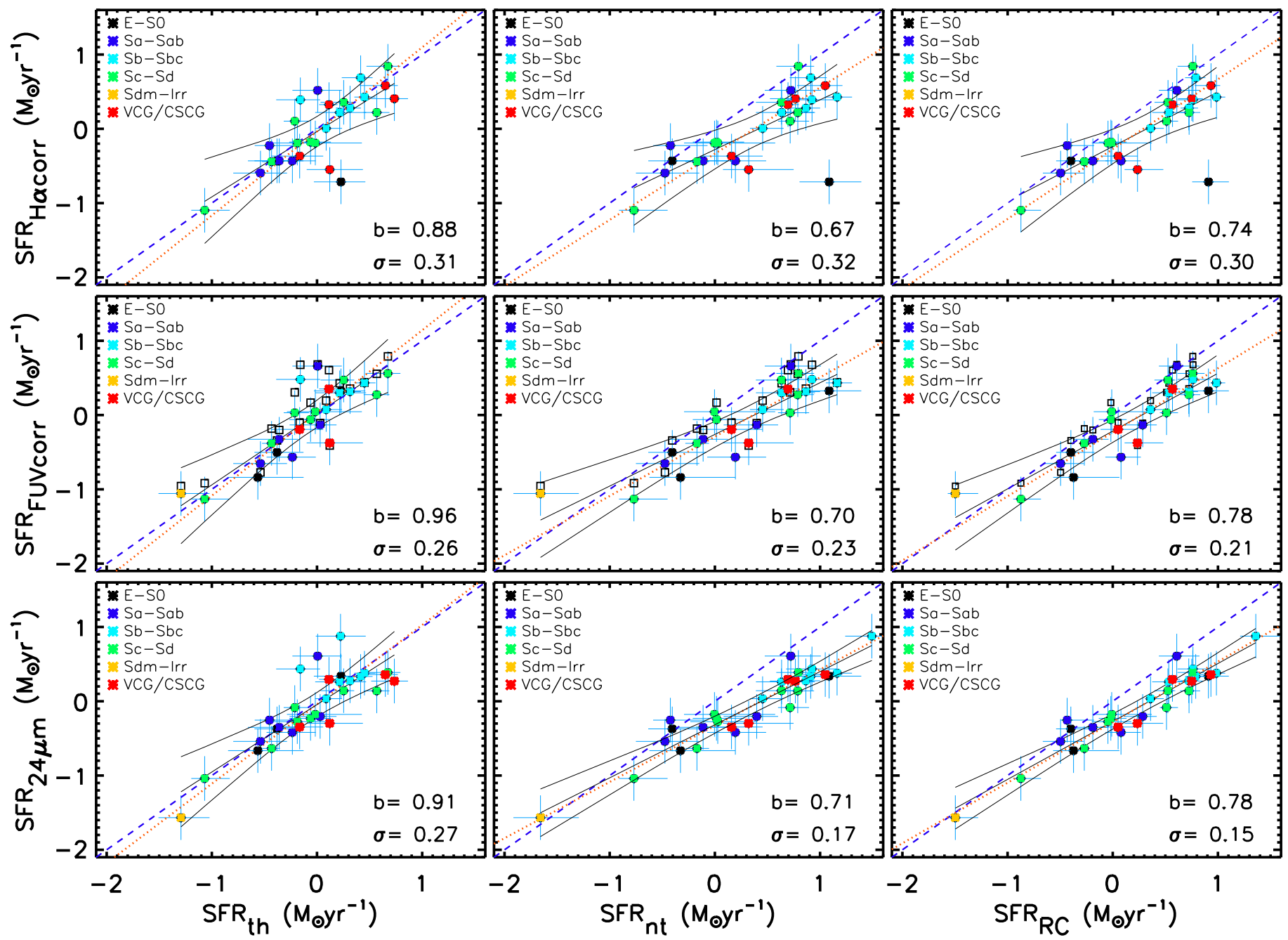

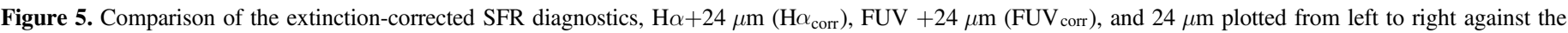

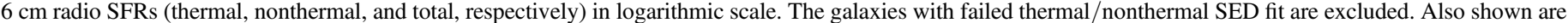

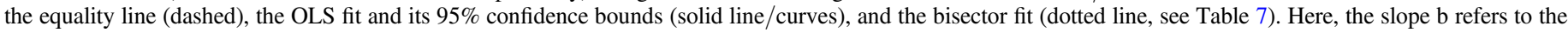

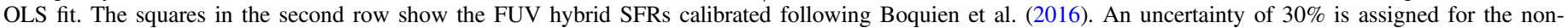
radio SFRs.

thermal, nonthermal, and total RC at $6 \mathrm{~cm}$. Falling within the $95 \%$ confidence bounds, the equality between the radio and non-radio SFRs is achieved best when using the thermal radio emission as the SFR tracer. Figure 5 also shows that the bisector fit (used in Table 7) is more robust to the outliers than the ordinary least square (OLS) fit, though they both agree regarding the uncertainties. The SFR is overestimated using the nonthermal radio (between $3 \%$ and $30 \%$, taking into account the errors) with respect to the non-radio SFRs. The nonthermal radio emission could, on the other hand, underestimate the local SFR in resolved studies because of diffusion of CREs (Murphy et al. 2011; Berkhuijsen et al. 2013). The tightest correlation holds between the $24 \mu \mathrm{m}$ and the nonthermal SFR, which hints at the nonthermal origin of the radio-IR correlation caused by a coupling between the gas and magnetic fields as shown in our resolved studies (Tabatabaei et al. 2013a, 2013c).

The uncertainties in the radio SFRs in Figure 5 are calculated using the error propagation technique accounting for the SED parameter errors and including the calibration, baselevel, and map fluctuation uncertainties. A $30 \%$ uncertainty is assumed for the non-radio SFRs. However, we caution that the uncertainty in the hybrid SFRs could be even larger. Taking into account contributions to the $24 \mu \mathrm{m}$ emission not associated with massive star formation, Leroy et al. (2008) found that the $24 \mu \mathrm{m}$ SFR estimators are systematically uncertain by a factor of $\sim 2$ leading to a calibration error of $50 \%$ for galaxy integrated SFRs based on the hybrid SFRs. We also note that correcting the FUV emission following Boquien et al. (2016) ${ }^{23}$ and Hao et al. (2011) leads to about similar SFRs, globally, considering the uncertainties.

As the next step, we investigate the use of the MRC bolometric radio luminosity, as a star-formation tracer. A tight correlation is found between the MRC and other SFR tracers $(r>0.8)$ among which we select the thermal radio emission as the ideal reference SFR tracer. The following relation holds between the thermal radio luminosity at $6 \mathrm{~cm}$ and the

\footnotetext{
$\overline{23}$ The FUV correction given by Boquien et al. (2016) was not applied to all galaxies due to either a lack of data or being out of the applicability bound.
} 

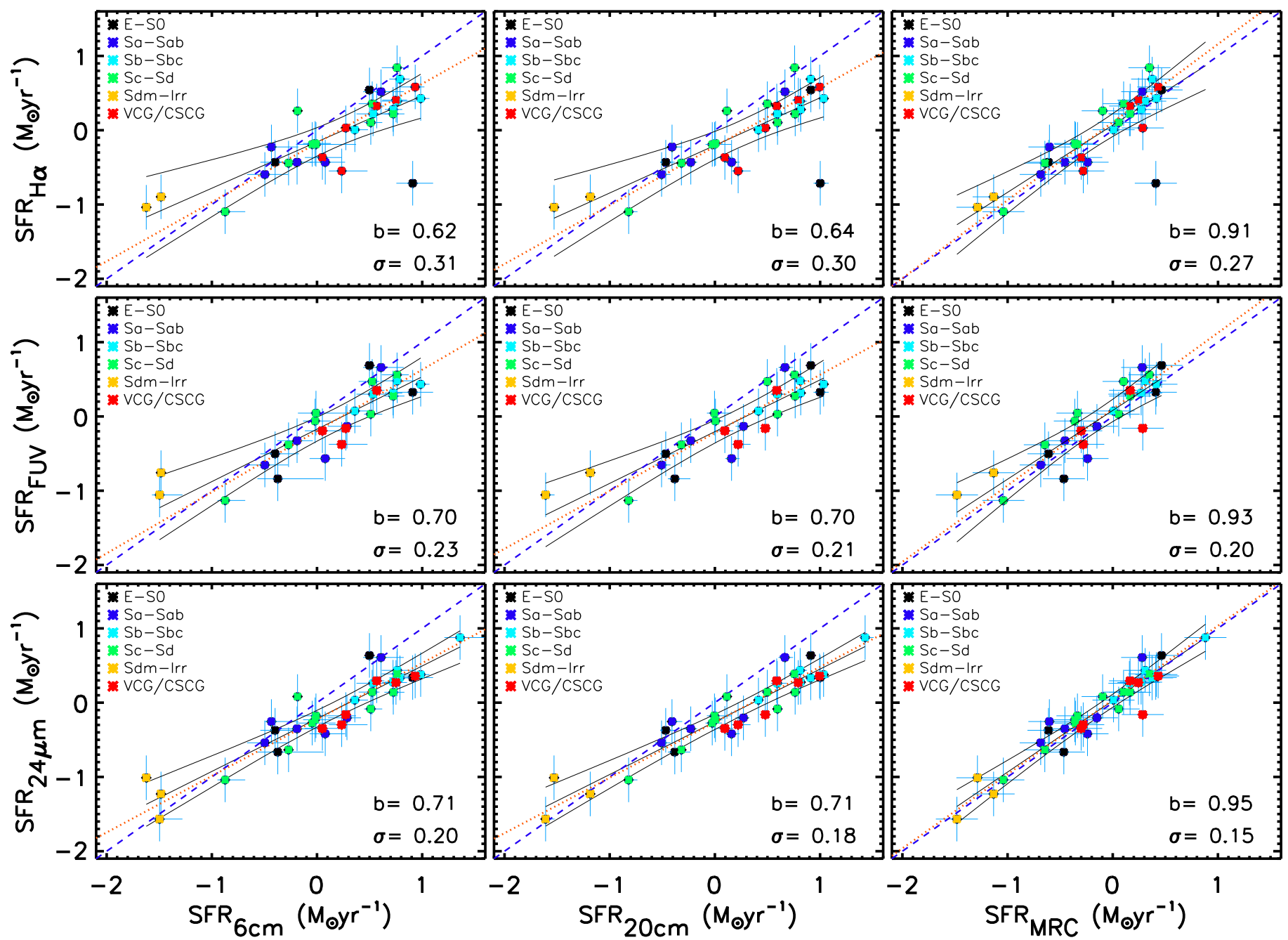

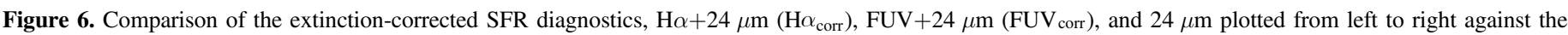

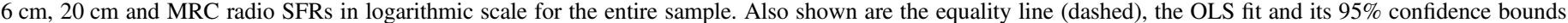

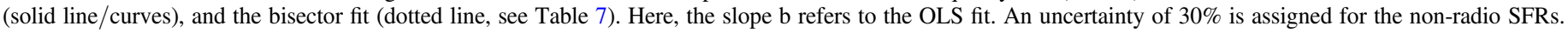

MRC,

$$
\begin{aligned}
& \log \left[\nu L_{\nu}^{\text {th }}(6 \mathrm{~cm})\right] \\
& \quad=(6.5 \pm 1.5)+(0.80 \pm 0.07) \log [\mathrm{MRC}] .
\end{aligned}
$$

The SRF calibration based on the MRC is hence derived using Equations (10) and (21),

$$
\left(\frac{\mathrm{SFR}_{\mathrm{MRC}}}{M_{\odot} \mathrm{yr}^{-1}}\right)=3.5 \times 10^{-31}\left(\frac{\mathrm{MRC}}{\mathrm{erg} \mathrm{s}^{-1}}\right)^{(0.80 \pm 0.07)},
$$

with a dispersion of $\simeq 0.2$ dex. Figure 6 shows that the non-radio SFRs agree better with SFR $_{M R C}$ than with those traced monochromatically in radio (i.e., $\mathrm{SFR}_{6 \mathrm{~cm}}$ and $\mathrm{SFR}_{20 \mathrm{~cm}}$ ). Moreover, using the MRC as an SFR tracer reduces the scatter $\sigma$ by $5 \%-30 \%$ with respect to the monochromatic radio SFR tracers. The fitted relations are given in Table 7.

\subsection{Calibration of MRC with Monochromatic Luminosities}

It would be useful to find simple relations that derive the MRC radio luminosity using a limited number of standard radio bands and applicable to a wider range of galaxy radio luminosities. This is particularly helpful when not enough data/frequencies are available. The following combination of the $6 \mathrm{~cm}(4.8 \mathrm{GHz})$ and $20 \mathrm{~cm}(1.4 \mathrm{GHz})$ bands ( $C$ and $L$ bands) recovers the radio $1-10 \mathrm{GHz}$ SED shapes,

$$
\mathrm{MRC}=\eta_{1} \nu L_{\nu}(20 \mathrm{~cm})+\eta_{2} \nu L_{\nu}(6 \mathrm{~cm}),
$$

with $\eta_{1}=0.32 \pm 0.02$ and $\eta_{2}=1.68 \pm 0.10$. The coefficients are derived from a singular value decomposition solution to an over-determined set of linear equations (Press et al. 1992). This relation reproduces the two-component model bolometric MRC luminosities to within $1 \%$ on average and a scatter of $8 \%$. For those galaxies with only single-component SED available, the model MRC and the above combination deviate by $13 \% \pm 5 \%$. We also emphasize that the combination given in Equation (23) resembles the model MRC better than a 
single band calibration (using either the $20 \mathrm{~cm}$ or $6 \mathrm{~cm}$ luminosity).

\section{Equipartition Magnetic Field}

The correlation between the nonthermal radio emission and the SFR tracers could show a connection between the magnetic field and star-formation activity. This is supported by the theory of amplification of magnetic fields by a small-scale turbulent dynamo (e.g., Gressel et al. 2008) occurring in star-forming regions. Assuming equipartition between cosmic rays and the magnetic field, theoretical studies suggest a relation between the magnetic field strength $B$ and the SFR $\left(B \sim \mathrm{SFR}^{0.3}\right.$, e.g., Schleicher \& Beck 2013). We investigate this dependency in the KINGFISHER sample.

As a by-product of the SED analysis, one can estimate the magnetic field strength. In case of equipartition between the energy densities of the magnetic field and cosmic rays $\left(\varepsilon_{\mathrm{CR}}=\varepsilon_{\mathrm{B}}=B^{2} / 8 \pi\right)$, the strength of the total magnetic field $B$ in Gauss is given by

$$
B=\left[\frac{4 \pi\left(2 \alpha_{\mathrm{nt}}+1\right) K^{\prime} I_{\mathrm{nt}} E_{\mathrm{p}}^{1-2 \alpha_{\mathrm{nt}}}\left(\frac{\nu}{2 c_{1}}\right)^{\alpha_{\mathrm{nt}}}}{\left(2 \alpha_{\mathrm{nt}}-1\right) c_{2} L c_{3}}\right]^{\frac{1}{\mathrm{n}_{\mathrm{n}+3}}}
$$

(Beck \& Krause 2005), where $K^{\prime}=K+1$ with $K$ the ratio between the number densities of cosmic-ray protons and electrons, $I_{\mathrm{nt}}$ is the nonthermal intensity in $\mathrm{erg} \mathrm{s}^{-1} \mathrm{~cm}^{-2} \mathrm{~Hz}^{-1} \mathrm{sr}^{-1}, L$ the pathlength through the synchrotron emitting medium in centimeters, and $\alpha_{\text {nt }}$ the mean synchrotron spectral index. $E_{\mathrm{p}}=938.26 \mathrm{MeV}=1.50 \times$ $10^{-3} \mathrm{erg}$ is the proton rest energy and

$$
\begin{aligned}
c_{1}= & 3 e /\left(4 \pi m_{\mathrm{e}}{ }^{3} c^{5}\right)=6.26428 \times 10^{18} \mathrm{erg}^{-2} \mathrm{~s}^{-1} \mathrm{G}^{-1}, \\
c_{2}\left(\alpha_{\mathrm{nt}}\right)= & \frac{1}{4} c_{4}\left(\alpha_{\mathrm{nt}}+5 / 3\right) /\left(\alpha_{\mathrm{nt}}+1\right) \Gamma\left[\left(3 \alpha_{\mathrm{nt}}+1\right) / 6\right] \\
& \times \Gamma\left[\left(3 \alpha_{\mathrm{nt}}+5\right) / 6\right], \\
c_{4}= & \sqrt{3} e^{3} /\left(4 \pi m_{e} c^{2}\right), \\
= & 1.86558 \times 10^{-23} \mathrm{erg} \mathrm{G}^{-1} \mathrm{sr}^{-1},
\end{aligned}
$$

with $\Gamma$ as the mathematical gamma function. For a region where the field is completely ordered and has a constant inclination $i$ with respect to the sky plane $\left(i=0^{\circ}\right.$ is the face-on view), $c_{3}=[\cos (i)]^{\left(\alpha_{\mathrm{nt}}+1\right)}$.

It is usually assumed that $K \simeq 100$ (Beck \& Krause 2005) and $L \simeq 1 \mathrm{kpc} / \cos i$. For $\alpha_{\mathrm{nt}}=1$, e.g., dominant synchrotron cooling of the CREs, and Equation (22) is reduced to $B=C\left(\frac{I_{\mathrm{nt}}}{\cos (i)}\right)^{1 / 4}$. Since we are working with flux density and not surface brightness, a more practical expression is

$$
B=B_{0}\left(\frac{\cos (i)}{\cos \left(i_{0}\right)}\right)^{-1 / 4}\left(\frac{S_{\mathrm{nt}}}{S_{\mathrm{nt}, 0}}\right)^{1 / 4},
$$

where $B_{0}$ is the magnetic field strength, $i_{0}$ is the inclination angle, and $S_{\mathrm{nt}}$ and $S_{\mathrm{nt}, 0}$ are the nonthermal flux of the target and a reference galaxy. We had determined $B$ for one of the KINGFISHER galaxies, NGC 6946, using Equation (24) in Tabatabaei et al. (2013c). This galaxy is used as the reference in Equation (25), i.e., $B_{0}=B_{\mathrm{N} 6946}=16 \mu \mathrm{G}$ and
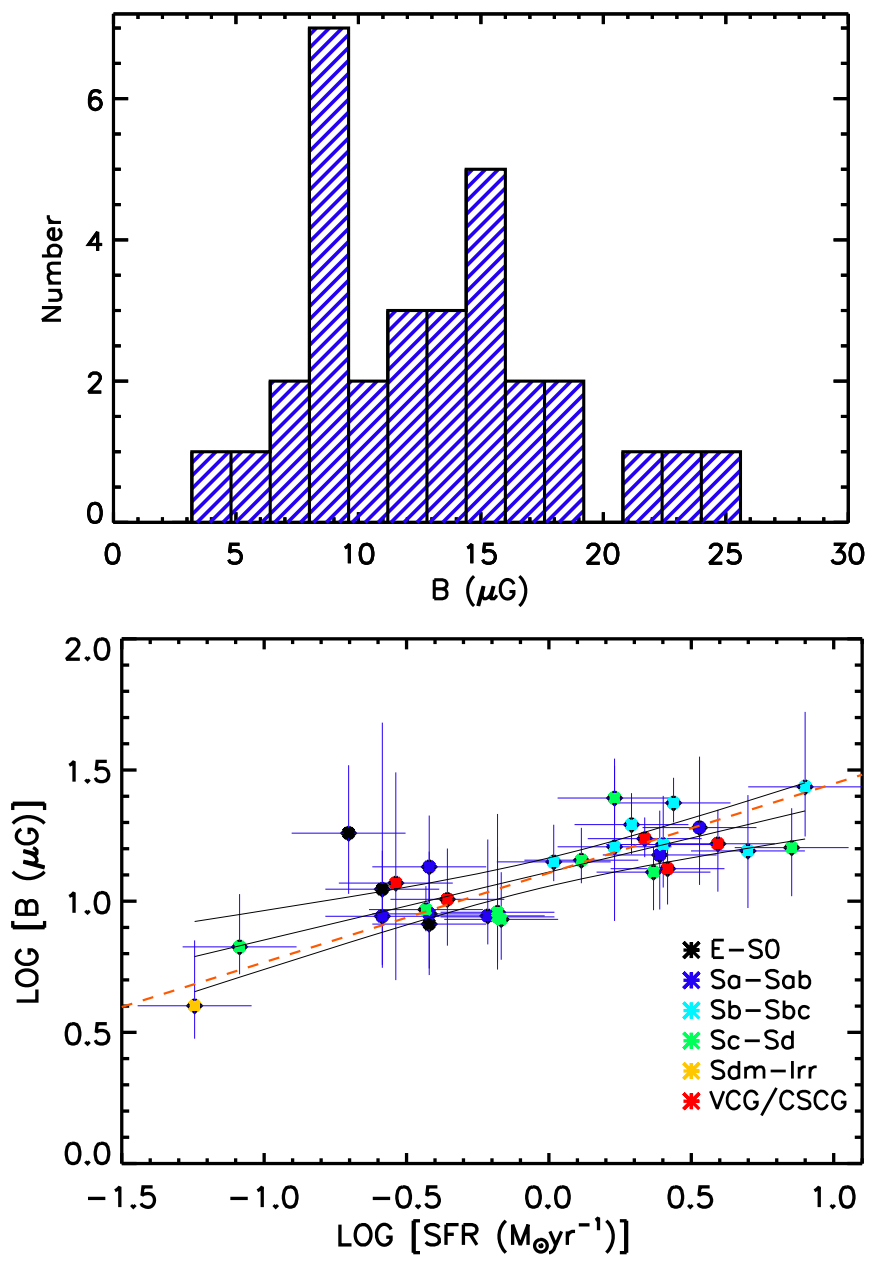

Figure 7. Top: distribution of the magnetic field strength in the KINGFISHER sample. Bottom: the magnetic field strength vs. the star-formation rate. Also shown are the OLS fit and its 95\% confidence bounds (solid line/curves) as well as the bisector fit (dashed line).

$S_{\mathrm{nt}, 0}=\left[S_{6.2 \mathrm{~cm}}^{4.8 \mathrm{GHz}}\left(1-f_{\mathrm{th}(6 \mathrm{~cm})}\right)\right]_{\mathrm{N} 6946}=0.5 \mathrm{Jy}$ (see Tables 4 and 5), and $i_{0}=33^{\circ}$, to estimate $B$ for other galaxies after correcting their fluxes for different distances. The magnetic field strength changes between $\simeq 4 \mu \mathrm{G}$ (IC 2574) and $27 \mu \mathrm{G}$ (NGC 2146) with a mean of $B=13.5 \pm 5.5 \mu \mathrm{G}$ in the KINGFISHER sample (see Figure 7-top). The $B$ values and their uncertainties, calculated using the error propagation technique, are listed in Table 4. Figure 7, bottom panel, shows that $B$ and SFR are correlated, $r=0.72 \pm 0.09$ and $^{24}$ $r_{s}=0.69 \pm 0.01$, as indicated first by the nonthermal radioSFR correlation (Section 5.1). The bisector fit shown in Figure 7 corresponds to

$$
\log B=(0.34 \pm 0.04) \log \mathrm{SFR}+(1.11 \pm 0.02),
$$

with $B$ in $\mu \mathrm{G}$ and SFR in $M_{\odot} \mathrm{yr}^{-1}$. The $B$-SFR dependency derived agrees with the theoretical proportionality $B \sim \mathrm{SFR}^{0.3}$ due to amplification of the turbulent magnetic field in starforming regions (Schleicher \& Beck 2013). Similar relations

\footnotetext{
${ }^{24}$ The formal error on the correlation coefficient depends on the strength of the correlation $r$ and the number of independent points $\mathrm{n}, \Delta r=\sqrt{1-r^{2}} / \sqrt{n-2}$ (Edwards 1979).
} 

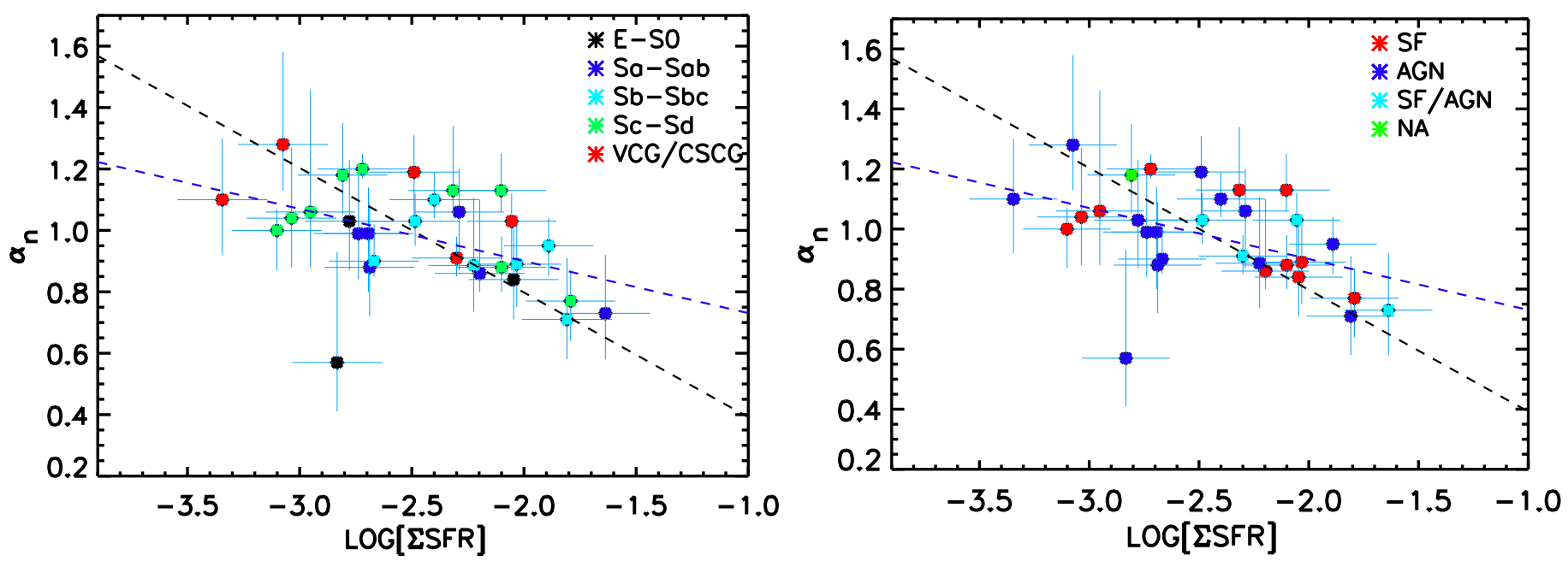

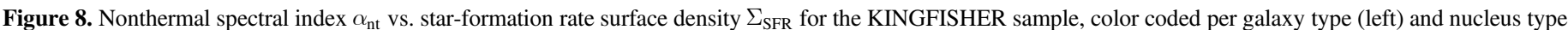

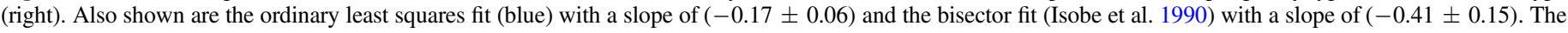

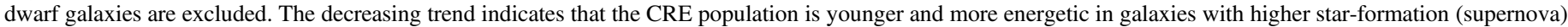
activity.

were found in observationally resolved studies (e.g., Chyży 2008; Chyży et al. 2011; Heesen et al. 2014). We emphasize that the nonthermal emission traces the total magnetic field that is a combination of the turbulent and ordered fields, and dominated by the turbulent field in starforming regions. Using the radio polarization data, instead, provides a more independent probe of the ordered large-scale magnetic field in galaxies. Our recent study in a sample of noninteracting/non-cluster galaxies shows that the ordered magnetic field is closely related to the rotation and the large-scale dynamics of galaxies (Tabatabaei et al. 2016).

\section{Further Discussion}

In this section, we investigate the dependencies of the radio SED parameters $\alpha_{\text {nt }}$ and $f_{\text {th }}$ on star formation and equipartition magnetic field. We also discuss the importance of this basic radio SED analysis for a better understanding of the observed IR-to-radio luminosity ratio in nearby galaxies leading to some hints for similar studies at high-z.

\subsection{The Influence of Star Formation on the Cosmic-Ray Electron Population}

After ejection from their sources in star-forming regions and propagating away, young CREs lose their energy through various cooling mechanisms: synchrotron, inverse Compton, Bremsstrahlung, and ionization. These cooling mechanisms change the energy index of CREs or equivalently the spectral index of the nonthermal emission $\alpha_{\mathrm{nt}}$ in different ways. Hence, $\alpha_{\mathrm{nt}}$ could change from galaxy to galaxy, depending on the balance between the young particles injected in star-forming regions and those cooled and aged in each galaxy (also see Basu et al. 2015a).

We obtained the SFR surface densities $\Sigma_{\text {SFR }}$ of the KINGFISHER galaxies using a non-radio SFR (the $\mathrm{H} \alpha+$ $24 \mu \mathrm{m}$ hybrid SFR) to avoid possible dependencies on the radio-SED parameters, and taking into account the optical size of the galaxies. Figure 8, left panel, shows a decrease in $\alpha_{\mathrm{nt}}$ with increasing $\Sigma_{\mathrm{SFR}}$ with a Pearson correlation coefficient of $r=-0.47 \pm 0.16$ and Spearman rank of $r_{s}=-0.51 \pm 0.01$ for normal galaxies $\left(\log (\mathrm{TIR})>8.9 L_{\odot}\right)$. The scatter increases when including the dwarfs and irregulars $(r=-0.42 \pm 0.16$ and $\left.r_{s}=-0.47 \pm 0.01\right)$. NGC 5866 appears as an outlier in Figure 8 due to its flat spectrum. Excluding it, the $\alpha_{\mathrm{nt}}-\Sigma_{\mathrm{SFR}}$ correlation is significantly enhanced $\left(r=r_{s}=-0.62 \pm 0.01\right)$.

Could the observed decreasing trend be partly due to AGNs? A flatter nonthermal spectrum in galaxies with higher $\Sigma_{\mathrm{SFR}}$ could occur due to the presence of flat spectrum AGNs. However, Figure 8, right panel, shows that the AGNs could not have a direct role on the observed trend, because the galaxies with AGNs could also have steep spectra (the two steepestspectrum galaxies actually host AGNs). Hence, the observed trend is mainly due to the SFR itself and not the AGNs.

Star formation could have an important influence on the energetics of the CRE population in a galaxy by increasing the number density of young and fresh relativistic particles with a flat spectrum via supernova explosions and their strong shocks. Even in SNRs, the observed nonthermal spectral index could be as flat as $\simeq 0.5-0.7$ (e.g., Berkhuijsen 1986). On the other other hand, the CREs in star-forming regions scatter off the very many pitch angles of the turbulent magnetic field (e.g., Chuvilgin \& Ptuskin 1993) to the surrounding medium with a diffusion length that is smaller for a smaller degree of field order (Tabatabaei et al. 2013a). This could lead to a high concentration of highenergy particles in turbulent star-forming regions causing CRE winds because of the local pressure gradient. They then escape with winds (see below) or are trapped in a weaker magnetic field far from star-forming regions and propagate/diffuse to larger scales producing diffuse synchrotron emission. Hence, starformation activities/feedback could flatten the global nonthermal spectrum in galaxies by (a) injecting young CREs with flat spectrum, (b) amplifying the turbulent magnetic field (Section 6) that helps the CREs to scatter off before they completely lose energy to synchrotron, and (c) producing strong winds and outflows that increase the convective escape probability of the CREs (e.g., Li et al. 2016). In this case, the CRE escape timescale is smaller than the synchrotron cooling timescale (for 
CREs with an isotropic pitch angle distribution, $t_{\mathrm{syn}}=\frac{24.57}{B^{2} \gamma} \mathrm{yr}$ with $B$ in Gauss and $\gamma$ the Lorentz factor). Hence, the global radio spectrum of more star-forming galaxies is dominated by radiation from younger CREs with flat spectra.

A flatter nonthermal spectrum in star-forming regions $\left(\alpha_{\mathrm{nt}}=0.5-0.7\right)$ than in the diffuse ISM $\left(\alpha_{\mathrm{nt}}>0.7\right)$ has already been found in resolved studies in M33 (Tabatabaei et al. 2007) and one of the KINGFISHER galaxies NGC 6946 (Tabatabaei et al. 2013c) for which high-resolution radio data were available. Detecting such an effect in global studies could, however, be complicated by contributions from various cooling mechanisms and inhomogenities that could induce scatter in the $\alpha_{\mathrm{nt}}-\Sigma_{\mathrm{SFR}}$ plane, as observed in Figure 8.

\subsection{The Influence of Magnetic Field on the Cosmic-Ray Electron Population}

Because the synchrotron emission depends on the magnetic field strength, it is also important to investigate the influence of $B$ on the energy spectrum of the CRE population. Theoretically, a positive correlation is expected due to increasing synchrotron cooling, a negative correlation for a CRE escape speed increasing with $B$, and no correlation due to other energy losses such as the bremsstrahlung loss. The positive correlation can be traced in the ISM far from star-forming regions, where the magnetic field is more uniform/ordered. The entangled/ turbulent field interrupts the continuous synchrotron cooling of the CREs and prevents further steepening of their emission spectrum by scattering them as occurs in star-forming regions (Section 7.1). For instance, in NGC 6946, the nonthermal spectrum along the ordered magnetic field is steeper than in the other ISM regions, particularly those with strong turbulent fields (Tabatabaei et al. 2013c). Hence, looking for a positive $\alpha_{\mathrm{nt}}-B$ correlation based on the integrated properties of the galaxies should be complicated by the presence of star-forming regions having low $\alpha_{\mathrm{nt}}$ and strong $B$ (which is mostly turbulent).

In our sample, we find a poor correlation with a rank of $r_{s}=-0.32 \pm 0.09$ at best (excluding the outliers, i.e., dwarfs and NGC 5866). The weakness of the correlation could be due to a combined effect from the star-forming and non-starforming ISM as discussed. The negative $r_{s}$ indicates the large influence from star-forming regions and the fact that $B$ is dominated by the turbulent magnetic field. Other cooling/ propagation effects could also cause complications in global studies.

\subsection{The Radio SED versus the IR SED}

Bolometric luminosities are a measure of the energy budget of galaxies emitting at certain ranges of frequencies. The IR bolometric luminosities have been studied in detail at various frequency intervals, e.g., TIR: $8-1000 \mu \mathrm{m}$ (Sanders \& Mirabel 1996), FIR: 42.5-122.5 $\mu \mathrm{m}$ (Rice et al. 1988), FIR: 40-500 $\mu \mathrm{m}$ (Chary \& Elbaz 2001), FIR/submm: 40$1000 \mu \mathrm{m}$ (Tabatabaei et al. 2013b), and TIR: 3-1100 $\mu \mathrm{m}$ (Galametz et al. 2013). For the KINGFISH sample, Dale et al. (2012) obtained the TIR (3-1100 $\mu \mathrm{m})$ luminosities using the Herschel and Spitzer data (see Table 1). To compare the emission energy budget of the galaxies in IR with that in radio, we must compare their IR and radio bolometric luminosities. However, to our knowledge, there is no definition of the radio bolometric luminosity over any frequency range in the literature apart from our current definition. Hence, Equation (6) serves as the only available definition of the bolometric luminosity in mid-radio MRC. We compare the SED of the IR and radio domains by means of the ratio of their integrated luminosities in two ways, (a) the TIR-to-MRC ratio:

$$
\langle q\rangle_{\mathrm{TIR}} \equiv \log \left(\frac{\mathrm{TIR}}{10^{3} \mathrm{MRC}}\right),
$$

and (b) the FIR-to-MRC ratio:

$$
\langle q\rangle_{\mathrm{FIR}} \equiv \log \left(\frac{\mathrm{FIR}}{10^{3} \mathrm{MRC}}\right),
$$

with TIR, FIR, and MRC luminosities in $\operatorname{erg~s}^{-1}$ (the MRC factor of $10^{3}$ in the denominator is selected arbitrarily so that $\langle q\rangle$ falls in the range of the $q$-parameter defined traditionally using the $20 \mathrm{~cm}$ radio luminosity, Helou et al. 1985). The FIR luminosities were obtained by integrating the KINGFISH SEDs (Dale et al. 2012) in the frequency interval 42-122 $\mu \mathrm{m}$. In the sample, $\langle q\rangle_{\text {TIR }}$ changes between 2.26 and 3.02 with a mean of $2.70 \pm 0.17$ (error is the scatter). The FIR-to-MRC ratio, $\langle q\rangle_{\mathrm{FIR}}$, changes between 1.7 and 4.2 with a mean of $2.37 \pm 0.36$.

The parameters $\langle q\rangle_{\mathrm{TIR}}$ and $\langle q\rangle_{\mathrm{FIR}}$ are useful to study the relative change in the IR and radio SEDs in terms of various astrophysical parameters. A first parameter is the SFR as an important energy source of both radio and IR emission. Figure 9, top panel, shows a likely decreasing trend of $\langle q\rangle$ versus SFR, particularly for SFR $>1 M_{\odot} \mathrm{yr}^{-1}$ with a Pearson correlation coefficient $r=-0.4 \pm 0.1$ for both cases. The Spearman rank coefficient is $r_{s}=-0.45 \pm 0.01$ for the $\langle q\rangle_{\mathrm{TIR}^{-}}$ SFR correlation, and $r_{s}=-0.42 \pm 0.02$ for the $\langle q\rangle_{\mathrm{FIR}}-\mathrm{SFR}$ correlation. Considering the thermal and nonthermal MRC separately in Equations (27) and (28), a clear anti-correlation is found for $\langle q\rangle$ versus SFR when using the nonthermal emission (Figure 9-middle). In this case, the Pearson correlation coefficient is $r=-0.5 \pm 0.1$ for both cases. The $\langle q\rangle$ based on the thermal emission is not correlated with SFR (Figure 9bottom). This shows that the nonthermal SED could be more sensitive to a change in massive star-formation activity than the thermal emission. One immediate cause could be the amplification of the magnetic fields in star-forming regions, adding more weight to the synchrotron emission, as shown in Section 6. As such, the observed weak anti-correlation may be due to the star-formation feedback inducing the magnetic field strength in galaxies (e.g., Pellegrini et al. 2009; Tabatabaei et al. 2015). This also explains the sublinear non-radio versus radio SFR correlations (also the famous IR-radio correlation) shown in Section 5.

\subsection{Implication for High-z Studies}

As the synchrotron emission is set by the magnetic field strength, Equation (24) implies a smaller FIR-to-nonthermal radio ratio with a higher rate of star formation in galaxies, as found already in Section 7.3 (see Figure 9). This also has an implication for high- $z$ studies: we expect to see a drop in the nonthermal part of $\langle q\rangle$ at high redshifts, where the more luminous/higher-star-forming objects are selected. However, most of the high- $z$ studies show either no evolution (e.g., Jarvis et al. 2010; Sargent et al. 2010) or only a tentatively slight 


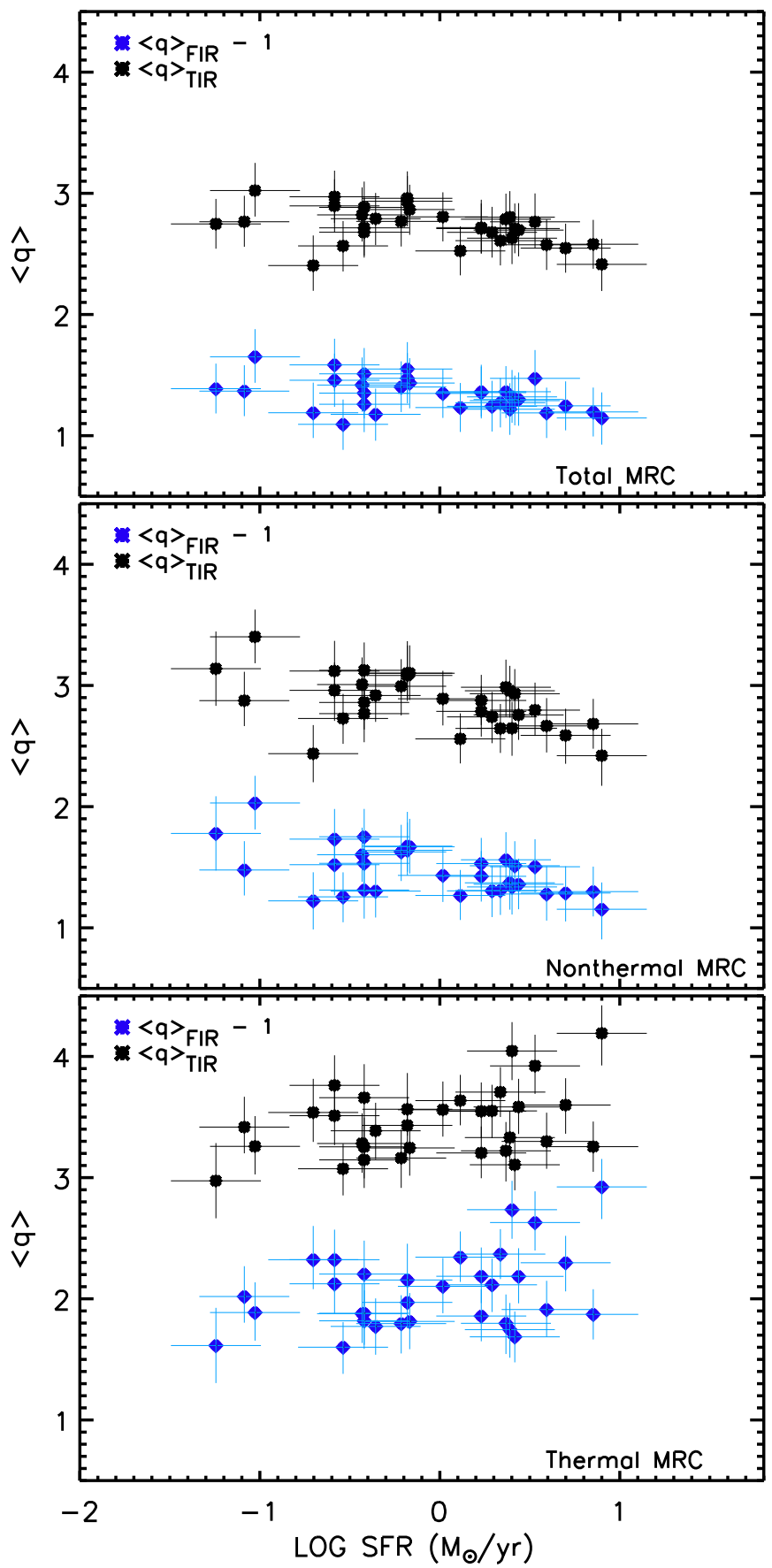

Figure 9. TIR-to-MRC ratio $\langle q\rangle_{\text {TIR }}$ and FIR-to-MRC ratio $\langle q\rangle_{\text {FIR }}$ vs. SFR for the RC (top) and its nonthermal (middle) and thermal components (bottom). A decreasing trend is indicated due to the nonthermal emission.

decrease of the IR to radio ratio ( $q_{\mathrm{IR}}$ with $z$, Ivison et al. 2010a, 2010b; Casey et al. 2012; Basu et al. 2015b). This could be of course due to the fact that no attempt is usually made to separate the thermal and nonthermal radio components when studying the IR-to-radio ratios.

Few high- $z$ studies have addressed variations of $q_{\mathrm{IR}}$ with dust temperature in galaxies leading to different results, i.e., either weak positive correlation (Magnelli et al. 2015) or a negative correlation (Ivison et al. 2010b; Smith et al. 2012). As shown in Figure 10, a correlation between $\langle q\rangle$ and the dust temperature,

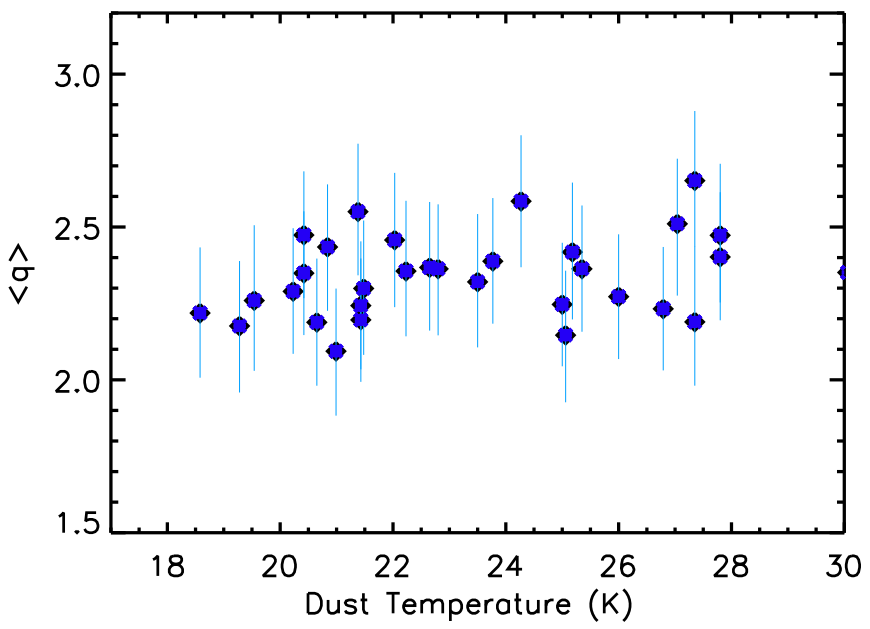

Figure 10. FIR-to-MRC radio ratio $\langle q\rangle_{\text {FIR }}$ vs. dust temperature.
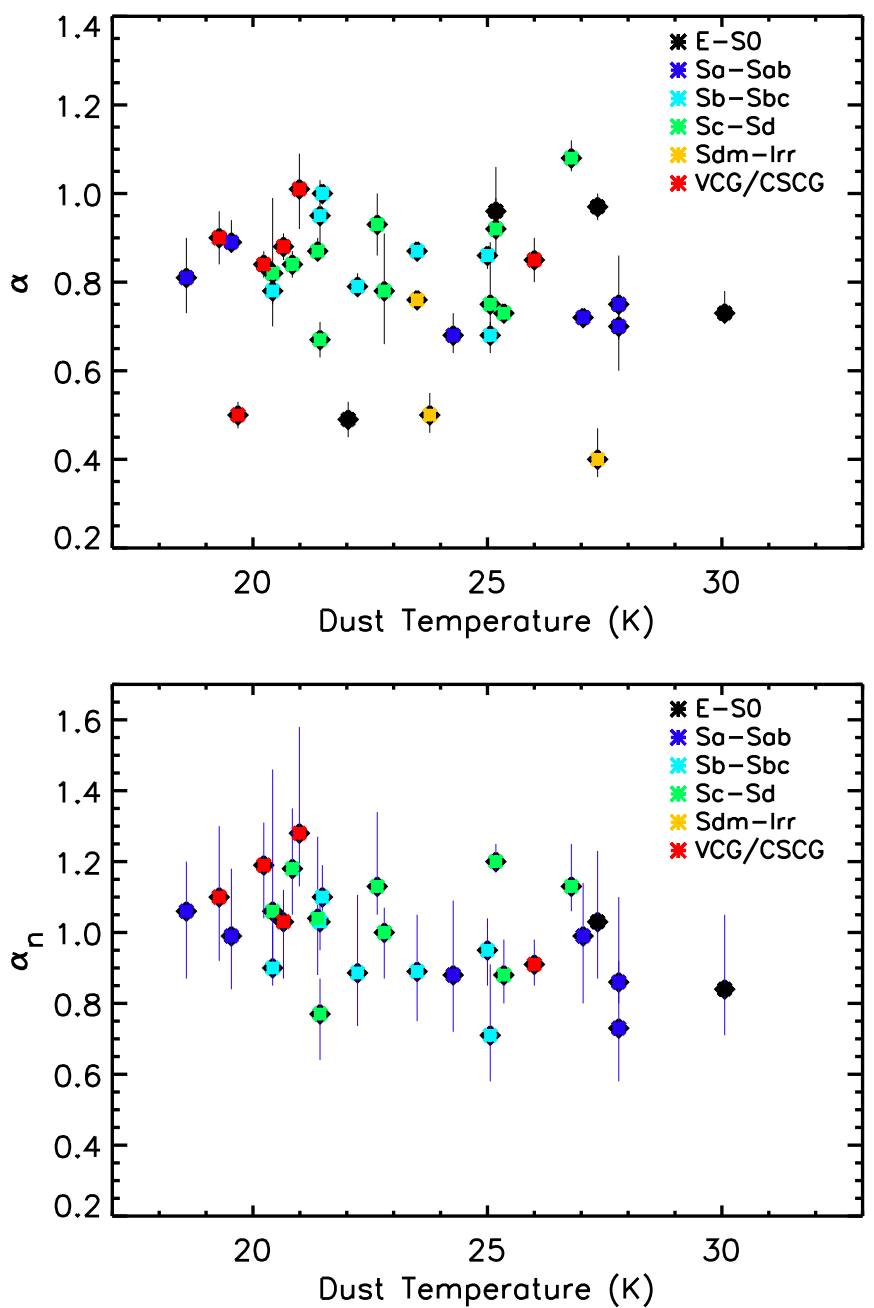

Figure 11. Top: the RC spectral index $\alpha$ vs. the dust temperature. Bottom: the nonthermal spectral index $\alpha_{\mathrm{nt}}$ exhibits a possible decreasing trend against the dust temperature excluding the flat radio sources $(\alpha<0.6)$.

derived by fitting a single modified black-body model to the IR SEDs (Dale et al. 2012), does not occur in nearby galaxies.

The radio spectral index was proposed as a redshift indicator for distant galaxies (Carilli \& Yun 1999), but the technique was 
Table 8

Thermal Fraction $f_{\text {th }}(6 \mathrm{~cm})$ Based on the Observed $\mathrm{H} \alpha, \mathrm{H} \alpha+24 \mu \mathrm{m}\left(\mathrm{H} \alpha_{\text {corr }}\right)$, and the Radio-SED Methods

\begin{tabular}{|c|c|c|c|}
\hline Galaxy & $\mathrm{H} \alpha$ & $\mathrm{H} \alpha_{\text {corr }}$ & radio-SED \\
\hline IC0342 & 0.17 & 0.27 & $\ldots$ \\
\hline IC2574 & 0.48 & 0.54 & $0.55_{0.12}^{0.14}$ \\
\hline NGC 0337 & 0.07 & 0.13 & $0.08_{0.03}^{0.09}$ \\
\hline NGC 0628 & 0.30 & 0.42 & $0.44_{0.12}^{0.11}$ \\
\hline NGC 1266 & 0.01 & 0.07 & $0.08_{0.20}^{0.15}$ \\
\hline NGC 1482 & 0.01 & 0.08 & $\ldots$ \\
\hline NGC 2146 & $\cdots$ & $\cdots$ & $0.20_{0.25}^{0.20}$ \\
\hline NGC 2798 & 0.03 & 0.10 & $0.07_{0.18}^{0.10}$ \\
\hline NGC 2841 & $\cdots$ & $\cdots$ & $0.22_{0.21}^{0.07}$ \\
\hline NGC 2976 & 0.23 & 0.32 & $0.27_{0.14}^{0.20}$ \\
\hline NGC 3049 & 0.23 & 0.44 & $0.31_{0.25}^{0.27}$ \\
\hline NGC 3184 & 0.27 & 0.39 & $0.39_{0.20}^{0.25}$ \\
\hline NGC 3190 & 0.05 & 0.11 & $0.18_{0.11}^{0.10}$ \\
\hline NGC 3265 & 0.08 & 0.29 & $0.33_{0.07}^{0.10}$ \\
\hline NGC 3521 & 0.10 & 0.18 & $0.15_{0.21}^{0.18}$ \\
\hline NGC 3627 & 0.08 & 0.18 & $0.16_{0.24}^{0.20}$ \\
\hline NGC 3938 & 0.24 & 0.34 & $0.28_{0.22}^{0.20}$ \\
\hline NGC 4236 & 0.36 & 0.42 & $\ldots$ \\
\hline NGC 4254 & 0.10 & 0.17 & $0.20_{0.14}^{0.09}$ \\
\hline NGC 4321 & 0.05 & 0.25 & $0.43_{0.20}^{0.07}$ \\
\hline NGC 4536 & 0.03 & 0.14 & $0.12_{0.04}^{0.06}$ \\
\hline NGC 4559 & 0.26 & 0.37 & $0.31_{0.30}^{0.25}$ \\
\hline NGC 4569 & 0.03 & 0.10 & $0.25_{0.18}^{0.15}$ \\
\hline NGC 4579 & 0.02 & 0.06 & $\ldots$ \\
\hline NGC 4631 & 0.07 & 0.11 & $0.23_{0.11}^{0.09}$ \\
\hline NGC 4725 & 0.18 & 0.25 & $0.25_{0.15}^{0.13}$ \\
\hline NGC 4736 & 0.04 & 0.21 & $0.25_{0.20}^{0.15}$ \\
\hline NGC 4826 & 0.04 & 0.26 & $0.30_{0.27}^{0.25}$ \\
\hline NGC 5055 & 0.05 & 0.15 & $0.17_{0.22}^{0.18}$ \\
\hline NGC 5457 & 0.11 & 0.25 & $0.20_{0.16}^{0.13}$ \\
\hline NGC 5713 & 0.01 & 0.10 & $0.04_{0.20}^{0.15}$ \\
\hline NGC 5866 & 0.09 & 0.13 & $0.15_{0.15}^{0.20}$ \\
\hline NGC 6946 & 0.20 & 0.29 & $0.24_{0.20}^{0.12}$ \\
\hline NGC 7331 & 0.05 & 0.15 & $0.12_{0.13}^{0.15}$ \\
\hline M51 & 0.04 & 0.10 & $0.15_{0.14}^{0.12}$ \\
\hline
\end{tabular}

shown to have limited accuracy (50\% redshift errors) due to a change in dust temperatures (Chapman et al. 2005). This also motivated us to look for any trend between the dust temperature and the radio spectral index in nearby galaxies, which could be used as a basic reference for high-z studies. Figure 11 shows no correlation between $\alpha$ and the dust temperature in our galaxies. On the other hand, a likely decreasing trend is found between $\alpha_{\text {nt }}$ versus the dust temperature $\left(r=-0.40 \pm 0.15\right.$ and $\left.r_{s}=-0.42 \pm 0.02\right)$. This can be explained by the positive correlation between the dust temperature and the star-formation surface density $\Sigma_{\text {SFR }}$ with about the same quality $(r \simeq+0.45)$, and considering that $\alpha_{\mathrm{nt}}$ decreases with $\Sigma_{\mathrm{SFR}}$ (see Section 4.1).

\section{Summary}

We compared the non-radio extinction-corrected diagnostics of SFRs with the radio SFRs for a sample of nearby galaxies, KINGFISHER, using both the MRC bolometric and monochromatic luminosities at 6 and $20 \mathrm{~cm}$. Our homogeneous and
Table 9

Thermal Fractions Predicted beyond the $1-10 \mathrm{GHz}$

\begin{tabular}{|c|c|c|c|c|c|}
\hline Galaxy & $350 \mathrm{MHz}$ & $15 \mathrm{GHz}$ & $22 \mathrm{GHz}$ & $33 \mathrm{GHz}$ & $45 \mathrm{GHz}$ \\
\hline IC0342 & $\ldots$ & $\ldots$ & $\ldots$ & $\ldots$ & $\ldots$ \\
\hline IC2574 & 0.11 & 0.75 & 0.81 & 0.86 & 0.90 \\
\hline NGC 0337 & 0.01 & 0.23 & 0.31 & 0.41 & 0.48 \\
\hline NGC 0628 & 0.05 & 0.55 & 0.60 & 0.68 & 0.73 \\
\hline NGC 1266 & 0.01 & 0.20 & 0.26 & 0.34 & 0.41 \\
\hline NGC 2146 & 0.00 & 0.03 & 0.04 & 0.05 & 0.06 \\
\hline NGC 2798 & 0.00 & 0.13 & 0.16 & 0.20 & 0.23 \\
\hline NGC 2841 & 0.03 & 0.56 & 0.64 & 0.73 & 0.78 \\
\hline NGC 2976 & 0.05 & 0.50 & 0.60 & 0.70 & 0.76 \\
\hline NGC 3049 & 0.01 & 0.45 & 0.53 & 0.60 & 0.65 \\
\hline NGC 3077 & $\ldots$ & $\ldots$ & $\ldots$ & $\ldots$ & $\ldots$ \\
\hline NGC 3184 & 0.05 & 0.60 & 0.68 & 0.76 & 0.81 \\
\hline NGC 3190 & 0.07 & 0.37 & 0.45 & 0.54 & 0.61 \\
\hline NGC 3265 & 0.03 & 0.57 & 0.64 & 0.70 & 0.75 \\
\hline NGC 3521 & 0.01 & 0.32 & 0.41 & 0.50 & 0.57 \\
\hline NGC 3627 & 0.01 & 0.31 & 0.37 & 0.45 & 0.51 \\
\hline NGC 3938 & 0.02 & 0.51 & 0.60 & 0.68 & 0.74 \\
\hline NGC 4236 & $\cdots$ & $\cdots$ & $\cdots$ & $\ldots$ & $\cdots$ \\
\hline NGC 4254 & 0.02 & 0.42 & 0.51 & 0.61 & 0.67 \\
\hline NGC 4321 & 0.03 & 0.73 & 0.81 & 0.87 & 0.90 \\
\hline NGC 4536 & 0.00 & 0.21 & 0.27 & 0.35 & 0.41 \\
\hline NGC 4559 & 0.04 & 0.68 & 0.76 & 0.84 & 0.88 \\
\hline NGC 4569 & 0.03 & 0.66 & 0.75 & 0.82 & 0.86 \\
\hline NGC 4579 & $\cdots$ & $\cdots$ & $\cdots$ & $\ldots$ & $\cdots$ \\
\hline NGC 4631 & 0.01 & 0.40 & 0.47 & 0.55 & 0.61 \\
\hline NGC 4725 & 0.03 & 0.53 & 0.63 & 0.72 & 0.78 \\
\hline NGC 4736 & 0.06 & 0.65 & 0.75 & 0.82 & 0.85 \\
\hline NGC 4826 & 0.04 & 0.50 & 0.57 & 0.65 & 0.70 \\
\hline NGC 5055 & 0.01 & 0.34 & 0.41 & 0.50 & 0.56 \\
\hline NGC 5457 & 0.02 & 0.42 & 0.50 & 0.59 & 0.65 \\
\hline NGC 5713 & 0.00 & 0.07 & 0.10 & 0.13 & 0.16 \\
\hline NGC 5866 & 0.00 & 0.20 & 0.23 & 0.27 & 0.30 \\
\hline NGC 6946 & 0.01 & 0.36 & 0.42 & 0.48 & 0.53 \\
\hline NGC 7331 & 0.01 & 0.33 & 0.42 & 0.52 & 0.60 \\
\hline M51 & 0.00 & 0.20 & 0.27 & 0.34 & 0.40 \\
\hline
\end{tabular}

careful analysis of the 1-10 GHz SEDs using new observations with the $100 \mathrm{~m}$ Effelsberg telescope allowed us to determine the MRC radio luminosities and the fractional contributions of the standard radio bands for the first time. The $1-10 \mathrm{GHz}$ bolometric luminosity is calibrated by a linear combination of the 6 and $20 \mathrm{~cm}$ bands (Equation (23)).

Unlike frequent assumptions, the nonthermal spectral index is not fixed. It changes over a wide range in the sample $(\sim 0.5-1.5$, Table 5), decreasing with increasing the starformation surface density of galaxies. This suggests the influence of star formation on the energetics of the CRE population, for example, by injecting high-energy cosmic rays. The average nonthermal spectral index derived for the $1-10 \mathrm{GHz}$ frequency range $\left(\alpha_{\mathrm{nt}}=0.97 \pm 0.16\right)$ is slightly steeper than that derived in the $400 \mathrm{MHz}-10 \mathrm{GHz}$ studies $(\simeq 0.8)$, considering the uncertainties. This difference could already indicate the low-frequency flattening of the synchrotron spectrum. Neglecting the thermal component, the $1-10 \mathrm{GHz}$ radio SEDs are fitted by a single power-law model with the mean spectral index of $\alpha=0.79 \pm 0.15$.

The thermal fraction changes from zero to $\sim 60 \%$ with a mean of $23 \%$ at $6 \mathrm{~cm}$, and from zero to $\sim 40 \%$ with a mean of $10 \%$ at $20 \mathrm{~cm}$ (Table 5) and agrees with the estimates based on the $\mathrm{H} \alpha$ methods (Table 8). It is the highest in dwarf irregular 
galaxies but does not show a clear correlation with morphology, $\Sigma_{\mathrm{SFR}}$, or metallicity.

We defined the mid-radio $(1-10 \mathrm{GHz})$ continuum bolometric luminosity, MRC, and obtained its distribution in the sample. The MRC luminosity of the KINGFISHER galaxies changes over $\sim 3$ orders of magnitude with a mean luminosity of $4.8 \times 10^{4} L_{\odot}$. Characterizing the average radio SED, we determined the contribution of the standard radio bands ( $L, S$, $C, X)$ in the mid-radio luminosity. We also presented a new calibration for the simple radio model (Condon et al. 1991), though large deviations could occur in individual galaxies.

Our study of the KINGFISHER sample, which includes a wide range of galaxy types, shows that the MRC is an ideal star-formation tracer. This is because of its good and linear correlation with other star-formation tracers including the FUV and $\mathrm{H} \alpha$ emission derived independently. We also presented SFR calibration relations using the MRC bolometric luminosity.

We found that the FIR-to-MRC luminosity ratio, $\langle q\rangle_{\mathrm{FIR}}$, could change with SFR that is due to the nonthermal component and its nonlinear correlation with SFR. Amplification of the equipartition turbulent magnetic fields in starforming regions could additionally strengthen the synchrotron power in galaxies with higher SFR, leading to a decrease in $\langle q\rangle$. Hence, star-formation feedback and magnetic fields could play a role in the balance between the radio and IR SEDs. Due to this feedback, the nonthermal radio emission overestimates the global SFR in starbursts and galaxies with high star-formation activity.

Extrapolating the SEDs beyond the $1-10 \mathrm{GHz}$, we predicted the thermal fractions at several frequencies from $350 \mathrm{MHz}$ to $45 \mathrm{GHz}$ (Table 9) based on the modeled SEDs. Comparing to the real observations at those selected frequencies it would be possible to determine the flattening of the SED (i.e., due to the free-free absorption of the synchrotron emission) at frequencies lower than $1 \mathrm{GHz}$, or contribution of the spinning dust emission at frequencies higher than $10 \mathrm{GHz}$.

We thank the anonymous referee for helpful comments. F.S.T. acknowledges support by the German Research Foundation DFG via the grant TA 801/1-1 and the Spanish Ministry of Economy and Competitiveness(MINECO) under grant number AYA2013-41243-P. We thank P. Muller, A. Kraus, E. Angelakis, I. Myserlis, and the Effelsberg staff for their help and support in observations and calibration of the cross-scan observations. R.B. acknowledges financial support from DFG Research Unit FOR1254. D.D.M acknowledges support from ERCStG 307215 (LODESTONE).

\section{Appendix A \\ Comparison with Other Thermal/Nonthermal Separation Techniques}

A degeneracy in parameter space occurs naturally when several free parameters are fitted simultaneously using the classical $\chi^{2}$ method. In the Bayesian MCMC approach, the confidence intervals are the most probable posteriors taken directly from the parameter space (see Figures 1). Hence the degeneracy is naturally included in the uncertainties $(16 \%-$ $84 \%$, equal-tailed intervals) reported in Table 5. To check further the reliability of the confidence intervals and the range of the uncertainties, we perform a comparison with a different thermal/nonthermal separation technique. The thermal radio emission can be optimally traced by the brightest hydrogen recombination line, the $\mathrm{H} \alpha$ emission, in galaxies after dereddening (Tabatabaei et al. 2007, 2013a, 2013c). In global studies, combining the $\mathrm{H} \alpha$ and the $24 \mu \mathrm{m}$ fluxes is used to deredden the $\mathrm{H} \alpha$ emission (Section 5.1). The thermal free-free emission traced based on this de-reddening could, however, be overestimated depending on the stellar population in a galaxy because the interstellar dust is not the only source of the $24 \mu \mathrm{m}$ emission (dusty atmospheres of carbon stars also emit the IR emission at $24 \mu \mathrm{m}$, e.g., Verley et al. 2009; Tabatabaei \& Berkhuijsen 2010; Boquien et al. 2016). The corrected $(\mathrm{H} \alpha+24 \mu \mathrm{m})$ and observed $\mathrm{H} \alpha$ fluxes can hence be used as upper-estimates and lower-estimates of the thermal radio flux, respectively. The following expression,

$$
S_{\mathrm{th}}^{\nu}=1.14 \times 10^{-14}\left(\frac{T_{e}}{10^{4} \mathrm{~K}}\right)^{0.34}\left(\frac{\nu}{\mathrm{GHz}}\right)^{-0.1} S_{\mathrm{H} \alpha},
$$

converts the $\mathrm{H} \alpha$ flux (corrected or observed) $S_{\mathrm{H} \alpha}$ in ergs s${ }^{-1} \mathrm{~cm}^{-2}$ to the thermal radio flux density $S_{\mathrm{th}}$ in erg s$^{-1} \mathrm{~cm}^{-2} \mathrm{~Hz}^{-1}$ at frequency $\nu$ (e.g., Deeg et al. 1997). We derive the thermal fraction at $6 \mathrm{~cm}, f_{\mathrm{th}}(6 \mathrm{~cm})=S_{\mathrm{th}}^{4.8 \mathrm{GHz}} /$ $S_{6 \mathrm{~cm}}^{4.8 \mathrm{GHz}}$ for the KINGFISHER galaxies with available $\mathrm{H} \alpha$ flux (Tabel 8). The thermal fraction based on the radio method (i.e., the median of the posterior PDFs in the Bayesian approach) mostly falls in between the two $\mathrm{H} \alpha$ estimates or is closer to the corrected $\mathrm{H} \alpha$ estimate. In few other cases, the radio and $\mathrm{H} \alpha$ estimates agree within the uncertainties. It is then worth noting that the Bayesian MCMC method is successful and reliable at capturing the correct answer, apart from the large degeneracy caused by the large observational errors taken from the literature (particularly the $10 \mathrm{GHz}$ data)—which in most cases widens the range of the uncertainties in the thermal fraction to non-physical, negative values.

\section{Appendix B \\ Plots of the SED Fitting with Bayesian MCMC}

The radio SED fits presented in Section 3 are shown in Figures 12-14.

\section{Appendix C The Radio SEDs Beyond 1-10 GHz}

By extrapolating the best-fit SED models, the synchrotron and free-free fluxes, and the thermal fractions can be estimated at frequencies higher and lower than $1-10 \mathrm{GHz}$. This may not be realistic due to the curvature of the synchrotron SED and its flattening at lower frequencies. In this case, the predicted fluxes are higher than the observed fluxes and they actually provide a basis for evaluating the flattening itself. On the other extreme, the high-frequency extrapolations could result in total flux densities that are lower than the observed fluxes, due to spinning dust emission (Bot et al. 2010) or magnetic nanoparticles (Draine \& Hensley 2012). Then, our predictions would help detect such emission in the sample. Table 9 lists the predicted thermal fractions at $350 \mathrm{MHz}, 15 \mathrm{GHz}, 22 \mathrm{GHz}$, $33 \mathrm{GHz}$, and $45 \mathrm{GHz}$ for each galaxy. 

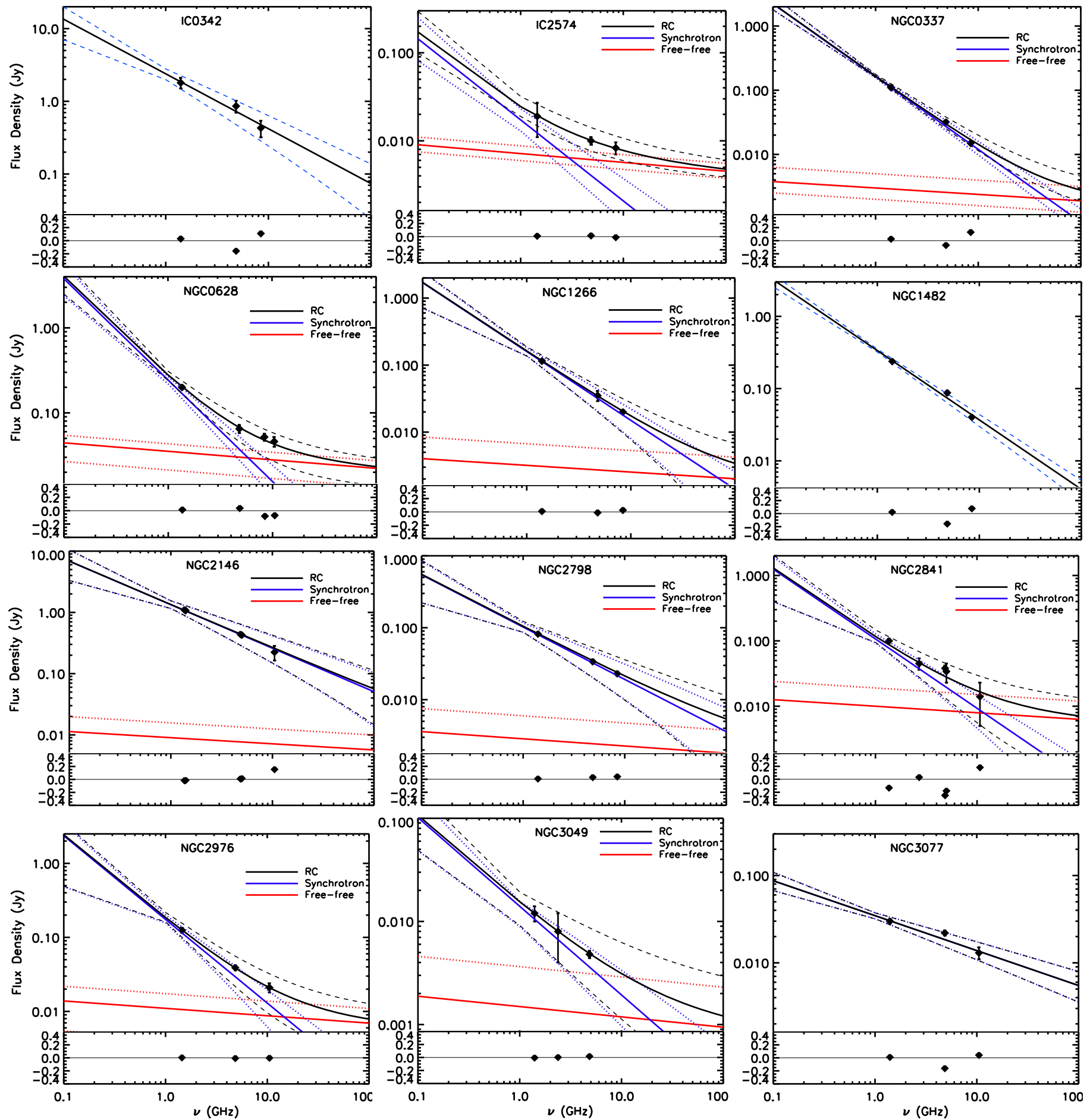

Figure 12. Radio SEDs (flux density vs. frequency) of the KINGFISHER sample (solid curves) for the total RC (black) and its nonthermal (blue) and thermal (red) components as well as their uncertainty curves (dashed for RC and dotted for its components). The points show the observed flux densities. Also shown are the relative residuals (modeled-observed/observed ratio) for each galaxy. 

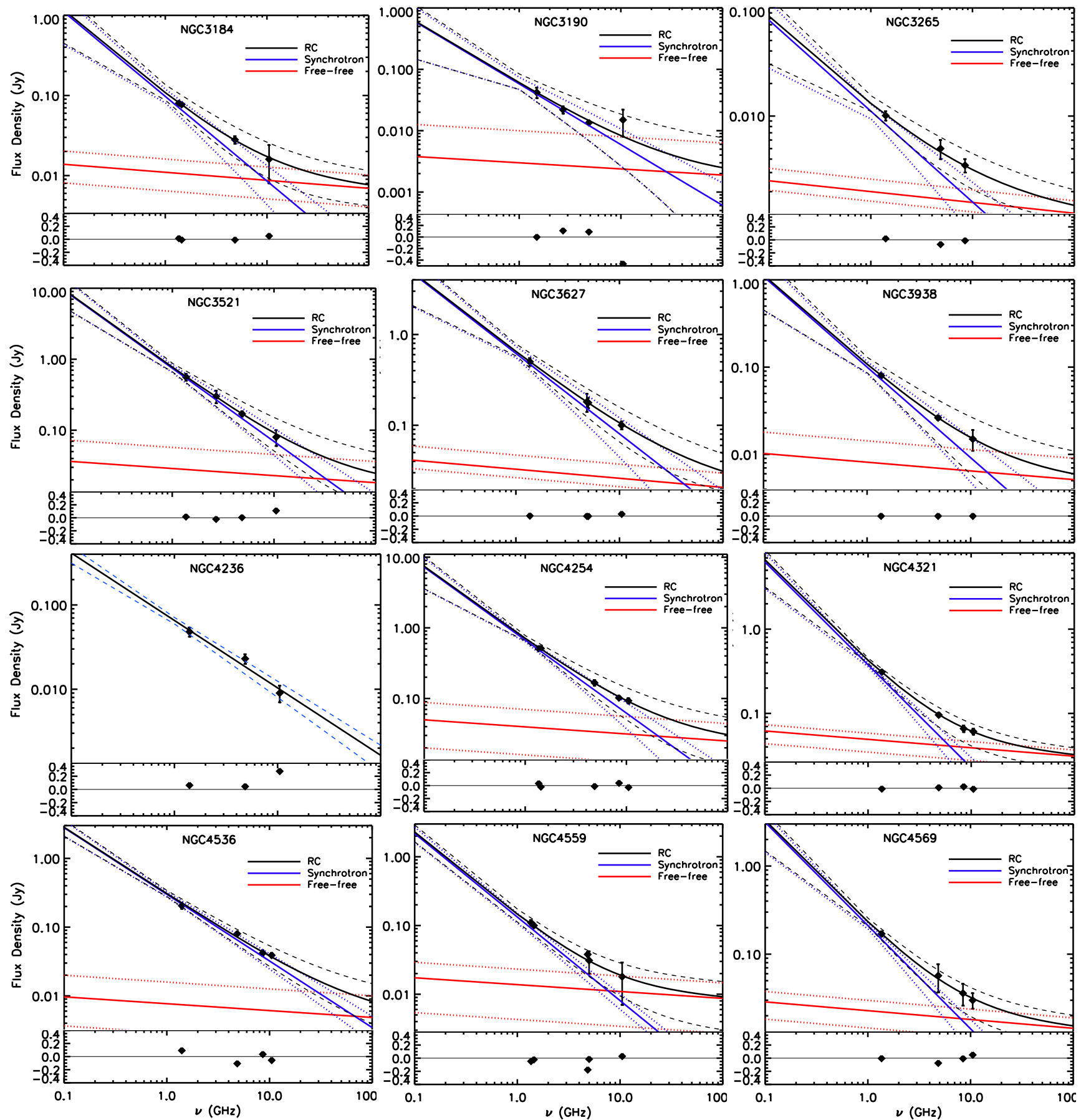

Figure 13. Same as Figure 12 for the rest of the sample. 

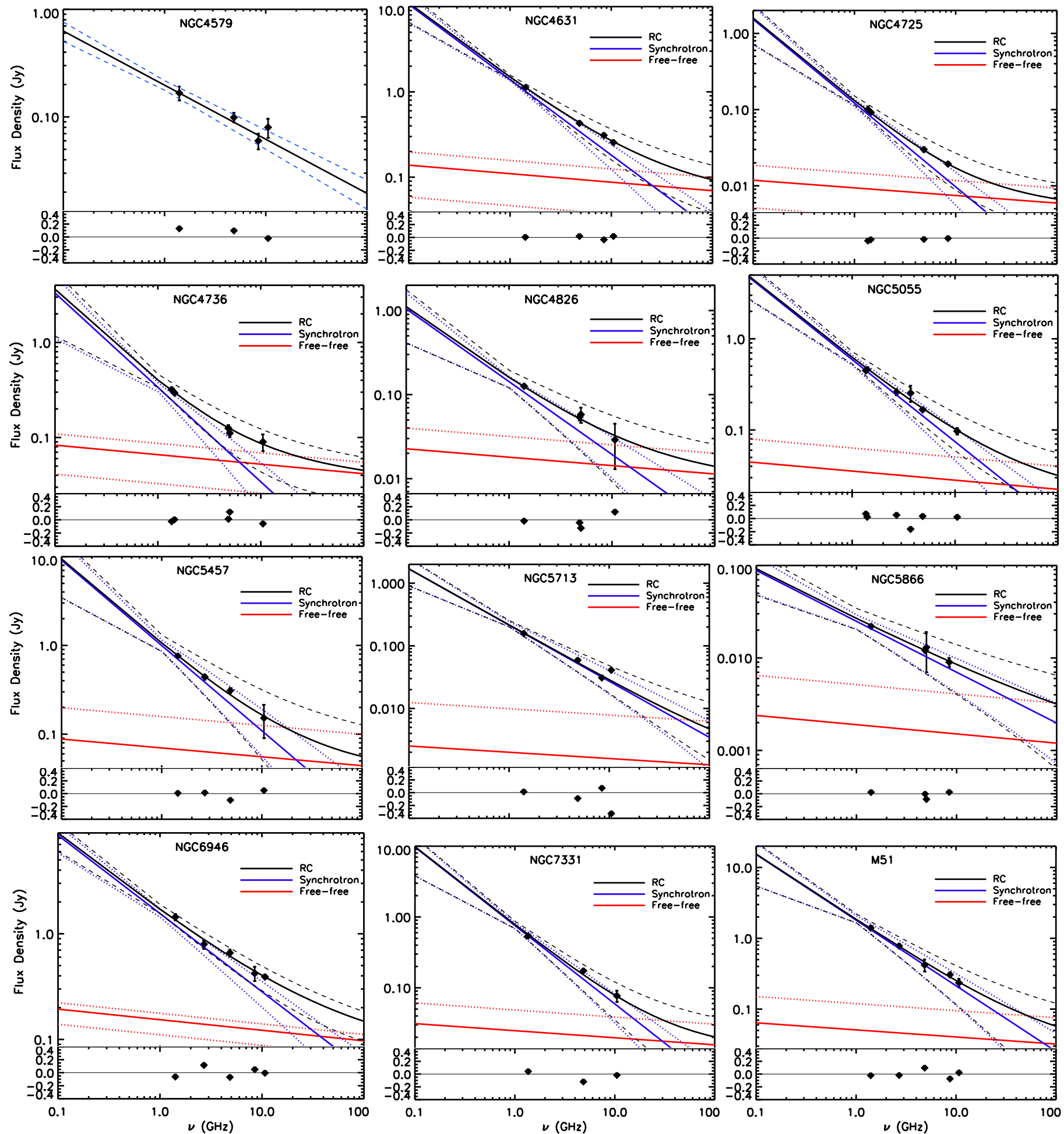

Figure 14. Same as Figures 12 and 13 for the rest of the sample.

\section{References}

Adebahr, B., Krause, M., Klein, U., et al. 2013, A\&A, 555, A23 Aniano, G., Draine, B. T., Calzetti, D., et al. 2012, ApJ, 756, 138 Baars, J. W. M., Genzel, R., Pauliny-Toth, I. I. K., \& Witzel, A. 1977, A\&A, 61, 99

Basu, A., Beck, R., Schmidt, P., \& Roy, S. 2015a, MNRAS, 449, 3879

Basu, A., Wadadekar, Y., Beelen, A., et al. 2015b, ApJ, 803, 51

Beck, R. 2015, A\&A, 578, A93
Beck, R., \& Krause, M. 2005, AN, 326, 414

Bell, E. F. 2003, ApJ, 586, 794

Berkhuijsen, E. M. 1986, A\&A, 166, 257

Berkhuijsen, E. M., Beck, R., \& Tabatabaei, F. S. 2013, MNRAS, 435, 1598 Berkhuijsen, E. M., Urbanik, M., Beck, R., \& Han, J. L. 2016, A\&A, 588, A114

Boquien, M., Kennicutt, R., Calzetti, D., et al. 2016, arXiv:1603.09340 Bot, C., Ysard, N., Paradis, D., et al. 2010, A\&A, 523, A20

Braun, R., Oosterloo, T. A., Morganti, R., Klein, U., \& Beck, R. 2007, A\&A, 461,455 
Brown, M. J. I., Jannuzi, B. T., Floyd, D. J. E., \& Mould, J. R. 2011, ApJL, 731, L41

Calzetti, D., Kennicutt, R. C., Engelbracht, C. W., et al. 2007, ApJ, 666, 870 Calzetti, D., Kennicutt, R. C., Jr., Bianchi, L., et al. 2005, ApJ, 633, 871

Calzetti, D., Wu, S.-Y., Hong, S., et al. 2010, ApJ, 714, 1256

Carilli, C. L., \& Yun, M. S. 1999, ApJL, 513, L13

Casey, C. M., Berta, S., Béthermin, M., et al. 2012, ApJ, 761, 140

Chapman, S. C., Blain, A. W., Smail, I., \& Ivison, R. J. 2005, ApJ, 622, 772

Chary, R., \& Elbaz, D. 2001, ApJ, 556, 562

Chuvilgin, L. G., \& Ptuskin, V. S. 1993, A\&A, 279, 278

Chyży, K. T. 2008, A\&A, 482, 755

Chyży, K. T., Bomans, D. J., Krause, M., et al. 2007a, A\&A, 462, 933

Chyży, K. T., Ehle, M., \& Beck, R. 2007b, A\&A, 474, 415

Chyży, K. T., Soida, M., Bomans, D. J., et al. 2006, A\&A, 447, 465

Chyży, K. T., Weżgowiec, M., Beck, R., \& Bomans, D. J. 2011, A\&A, 529, A94

Ciardullo, R., Rubin, V. C., Ford, W. K., Jr., Jacoby, G. H., \& Ford, H. C. 1988, AJ, 95, 438

Colombo, D., Meidt, S. E., Schinnerer, E., et al. 2014, ApJ, 784, 4

Condon, J. J. 1992, ARA\&A, 30, 575

Condon, J. J., Cotton, W. D., \& Broderick, J. J. 2002, AJ, 124, 675

Condon, J. J., Cotton, W. D., Greisen, E. W., et al. 1998, AJ, 115, 1693

Condon, J. J., Huang, Z.-P., Yin, Q. F., \& Thuan, T. X. 1991, ApJ, 378, 65

Condon, J. J., \& Yin, Q. F. 1990, ApJ, 357, 97

da Cunha, E., Charlot, S., \& Elbaz, D. 2008, MNRAS, 388, 1595

Dale, D. A., Aniano, G., Engelbracht, C. W., et al. 2012, ApJ, 745, 95

Dale, D. A., Gil de Paz, A., Gordon, K. D., et al. 2007, ApJ, 655, 863

Deeg, H.-J., Duric, N., \& Brinks, E. 1997, A\&A, 323, 323

Draine, B. T., \& Hensley, B. 2012, ApJ, 757, 103

Dressel, L. L., \& Condon, J. J. 1978, ApJS, 36, 53

Dumas, G., Schinnerer, E., Tabatabaei, F. S., et al. 2011, AJ, 141, 41

Duric, N., Bourneuf, E., \& Gregory, P. C. 1988, AJ, 96, 81

Edwards, A. L. 1979, Multiple Regression and Analysis of Variance and Covariance (San Francisco, CA: W.H. Freeman and Company)

Ehle, M., \& Beck, R. 1993, A\&A, 273, 45

Emerson, D. T., \& Graeve, R. 1988, A\&A, 190, 353

Emerson, D. T., Klein, U., \& Haslam, C. G. T. 1979, A\&A, 76, 92

Foreman-Mackey, D., Hogg, D. W., Lang, D., \& Goodman, J. 2013, PASP, 125,306

Galametz, M., Kennicutt, R. C., Calzetti, D., et al. 2013, MNRAS, 431, 1956

Gil de Paz, A., Boissier, S., Madore, B. F., et al. 2007, ApJS, 173, 185

Gioia, I. M., \& Fabbiano, G. 1987, ApJS, 63, 771

Gioia, I. M., Gregorini, L., \& Klein, U. 1982, A\&A, 116, 164

Gressel, O., Elstner, D., Ziegler, U., \& Rüdiger, G. 2008, A\&A, 486, L35

Griffin, M. J., Abergel, A., Abreu, A., et al. 2010, A\&A, 518, L3

Griffith, M. R., Wright, A. E., Burke, B. F., \& Ekers, R. D. 1994, ApJS, 90, 179

Griffith, M. R., Wright, A. E., Burke, B. F., \& Ekers, R. D. 1995, ApJS, 97, 347

Hao, C.-N., Kennicutt, R. C., Johnson, B. D., et al. 2011, ApJ, 741, 124

Harnett, J. I., Beck, R., \& Buczilowski, U. R. 1989, A\&A, 208, 32

Haslam, C. G. T. 1974, A\&AS, 15, 333

Heesen, V., Brinks, E., Leroy, A. K., et al. 2014, AJ, 147, 103

Helou, G., Soifer, B. T., \& Rowan-Robinson, M. 1985, ApJL, 298, L7

Hummel, E., van der Hulst, J. M., \& Dickey, J. M. 1984, A\&A, 134, 207

Hunt, L. K., Draine, B. T., Bianchi, S., et al. 2015, A\&A, 576, A33

Isobe, T., Feigelson, E. D., Akritas, M. G., \& Babu, G. J. 1990, ApJ, 364, 104

Israel, F. P., \& van der Hulst, J. M. 1983, AJ, 88, 1736

Ivison, R. J., Alexander, D. M., Biggs, A. D., et al. 2010a, MNRAS, 402, 245

Ivison, R. J., Magnelli, B., Ibar, E., et al. 2010b, A\&A, 518, L31

Jarvis, M. J., Smith, D. J. B., Bonfield, D. G., et al. 2010, MNRAS, 409, 92

Jurusik, W., Drzazga, R. T., Jableka, M., et al. 2014, A\&A, 567, A134

Kennicutt, R. C., Calzetti, D., Aniano, G., et al. 2011, PASP, 123, 1347

Kennicutt, R. C., Jr. 1998, ARA\&A, 36, 189

Kennicutt, R. C., Jr., Armus, L., Bendo, G., et al. 2003, PASP, 115, 928

Kennicutt, R. C., Jr, \& Evans, N. J., II 2012, arXiv:1204.3552

Kennicutt, R. C., Jr., Hao, C.-N., Calzetti, D., et al. 2009, ApJ, 703, 1672
Klein, U., \& Emerson, D. T. 1981, A\&A, 94, 29

Klein, U., Wielebinski, R., \& Beck, R. 1984, A\&A, 135, 213

Lacki, B. C., \& Beck, R. 2013, MNRAS, 430, 3171

Lacki, B. C., Thompson, T. A., \& Quataert, E. 2010, ApJ, 717, 1

Leroy, A. K., Walter, F., Brinks, E., et al. 2008, AJ, 136, 2782

Li, J.-T., Beck, R., Dettmar, R.-J., et al. 2016, MNRAS, 456, 1723

Loiseau, N., Klein, U., Greybe, A., Wielebinski, R., \& Haynes, R. F. 1987, A\&A, 178, 62

Longair, M. S. 1994, High Energy Astrophysics, Vol. 2: Stars, the Galaxy and the Interstellar Medium (2nd ed.; Cambridge: Cambridge Univ. Press)

Lu, N., Bendo, G. J., Boselli, A., et al. 2014, ApJ, 797, 129

Magnelli, B., Ivison, R. J., Lutz, D., et al. 2015, A\&A, 573, A45

Marvil, J., Owen, F., \& Eilek, J. 2015, AJ, 149, 32

Matsushita, S., Sakamoto, K., Kuo, C.-Y., et al. 2004, ApJL, 616, L55

Mora, S. C., \& Krause, M. 2013, A\&A, 560, A42

Mulcahy, D. D., Horneffer, A., Beck, R., et al. 2014, A\&A, 568, A74

Murphy, E. J., Condon, J. J., Schinnerer, E., et al. 2011, ApJ, 737, 67

Murphy, E. J., Kenney, J. D. P., Helou, G., Chung, A., \& Howell, J. H. 2009, ApJ, 694, 1435

Nicholls, D. C., Dopita, M. A., Sutherland, R. S., Jerjen, H., \& Kewley, L. J. 2014, ApJ, 790, 75

Niklas, S., Klein, U., Braine, J., \& Wielebinski, R. 1995, A\&AS, 114, 21

Niklas, S., Klein, U., \& Wielebinski, R. 1997, A\&A, 322, 19

Pellegrini, E. W., Baldwin, J. A., Ferland, G. J., Shaw, G., \& Heathcote, S. 2009, ApJ, 693, 285

Poglitsch, A., Waelkens, C., Geis, N., et al. 2010, A\&A, 518, L2

Press, W. H., Teukolsky, S. A., Vetterling, W. T., \& Flannery, B. P. 1992, Numerical Recipes in FORTRAN. The Art of Scientific Computing (2nd ed.; Cambridge: Cambridge Univ. Press)

Relaño, M., Lisenfeld, U., Pérez-González, P. G., Vílchez, J. M., \& Battaner, E. 2007, ApJL, 667, L141

Rice, W., Lonsdale, C. J., Soifer, B. T., et al. 1988, ApJS, 68, 91

Rieke, G. H., Alonso-Herrero, A., Weiner, B. J., et al. 2009, ApJ, 692, 556

Rujopakarn, W., Rieke, G. H., Weiner, B. J., et al. 2013, ApJ, 767, 73

Sanders, D. B., \& Mirabel, I. F. 1996, ARA\&A, 34, 749

Sargent, M. T., Schinnerer, E., Murphy, E., et al. 2010, ApJL, 714, L190

Schleicher, D. R. G., \& Beck, R. 2013, A\&A, 556, A142

Schmitt, H. R., Calzetti, D., Armus, L., et al. 2006, ApJS, 164, 52

Smith, M. W. L., Eales, S. A., Gomez, H. L., et al. 2012, ApJ, 756, 40

Sofue, Y., \& Reich, W. 1979, A\&AS, 38, 251

Sramek, R. 1975, AJ, 80, 771

Srivastava, S., Kantharia, N. G., Basu, A., Srivastava, D. C., \& Ananthakrishnan, S. 2014, MNRAS, 443, 860

Stauffer, J. R. 1982, ApJ, 262, 66

Stil, J. M., Krause, M., Beck, R., \& Taylor, A. R. 2009, ApJ, 693, 1392

Tabatabaei, F., Braine, J., Kramer, C., et al. 2015, in Proc. of The Many Facets of Extragalactic Radio Surveys: Towards New Scientific Challenges, Star Formation (Trieste: SISSA), 14

Tabatabaei, F. S., Beck, R., Krügel, E., et al. 2007, A\&A, 475, 133

Tabatabaei, F. S., \& Berkhuijsen, E. M. 2010, A\&A, 517, 77

Tabatabaei, F. S., Berkhuijsen, E. M., Frick, P., Beck, R., \& Schinnerer, E. 2013a, A\&A, 557, 129

Tabatabaei, F. S., Martinsson, T. P. K., Knapen, J. H., et al. 2016, ApJL, 818, L10

Tabatabaei, F. S., Schinnerer, E., Murphy, E. J., et al. 2013c, A\&A, 552, 19

Tabatabaei, F. S., Weiß, A., Combes, F., et al. 2013b, A\&A, 555, 128

Tammann, G. A. 1982, in Supernovae: A Survey of Current Research (NATO ASIC Proc. 90), ed. M. J. Rees \& R. J. Stoneham (Dordrecht: Reidel), 371

Verley, S., Corbelli, E., Giovanardi, C., \& Hunt, L. K. 2009, A\&A, 493, 453

Vollmer, B., Thierbach, M., \& Wielebinski, R. 2004, A\&A, 418, 1

Weżgowiec, M., Urbanik, M., Beck, R., Chyży, K. T., \& Soida, M. 2012, A\&A, 545, A69

White, R. L., \& Becker, R. H. 1992, ApJS, 79, 331

Wu, H., Cao, C., Hao, C.-N., et al. 2005, ApJL, 632, L79

Xu, C. 1990, ApJL, 365, L47 